\title{
Efficient methodologies for system matrix modelling in iterative image reconstruction for rotating high-resolution PET
}

\author{
J E Ortuño ${ }^{1,2}$, G Kontaxakis ${ }^{1,2}$, J L Rubio ${ }^{1,2}$, P Guerra $^{2}$ and A Santos ${ }^{1,2}$ \\ ${ }^{1}$ Departamento de Ingeniería Electrónica (DIE), Universidad Politécnica de Madrid, Ciudad \\ Universitaria s/n, 28040 Madrid, Spain \\ ${ }^{2}$ Networking Research Center on Bioengineering, Biomaterials and Nanomedicine \\ (CIBER-BBN), Madrid, Spain \\ E-mail: juanen@die.upm.es
}

Received 23 September 2009, in final form 22 December 2009

Published 12 March 2010

Online at stacks.iop.org/PMB/55/1833

\begin{abstract}
A fully 3D iterative image reconstruction algorithm has been developed for high-resolution PET cameras composed of pixelated scintillator crystal arrays and rotating planar detectors, based on the ordered subsets approach. The associated system matrix is precalculated with Monte Carlo methods that incorporate physical effects not included in analytical models, such as positron range effects and interaction of the incident gammas with the scintillator material. Custom Monte Carlo methodologies have been developed and optimized for modelling of system matrices for fast iterative image reconstruction adapted to specific scanner geometries, without redundant calculations. According to the methodology proposed here, only one-eighth of the voxels within two central transaxial slices need to be modelled in detail. The rest of the system matrix elements can be obtained with the aid of axial symmetries and redundancies, as well as in-plane symmetries within transaxial slices. Sparse matrix techniques for the non-zero system matrix elements are employed, allowing for fast execution of the image reconstruction process. This $3 \mathrm{D}$ image reconstruction scheme has been compared in terms of image quality to a $2 \mathrm{D}$ fast implementation of the OSEM algorithm combined with Fourier rebinning approaches. This work confirms the superiority of fully 3D OSEM in terms of spatial resolution, contrast recovery and noise reduction as compared to conventional 2D approaches based on rebinning schemes. At the same time it demonstrates that fully 3D methodologies can be efficiently applied to the image reconstruction problem for high-resolution rotational PET cameras by applying accurate pre-calculated system models and taking advantage of the system's symmetries.
\end{abstract}




\section{Introduction}

Positron emission tomography (PET) is a powerful functional imaging technique that is nowadays widely applied in human clinical practice, primarily in the field of oncology, as well as in cardiology, neurology, etc. PET is also used regularly in pre-clinical (i.e. small animal) research and drug development, and dedicated small animal scanners have become increasingly popular. The challenge for high-resolution small animal PET scanners and other reduced field dedicated scanners is to achieve fast and accurate image reconstruction procedures in target volumes that are orders of magnitude smaller than the size of the human body. High-resolution tomographs are designed to optimize spatial resolution while maintaining acceptable detection sensitivity. They usually consist of a set of detector modules arranged in a static ring (Surti et al 2005, Tai et al 2005, Wang et al 2006) or mounted on a rotating gantry (Del Guerra et al 2006, Rouze and Hutchins 2003). The high-resolution detector modules are made of pixelated scintillator crystals coupled to position-sensitive photomultipliers (PSPMT) (Del Guerra et al 2006, Huisman et al 2007, Tai et al 2005, Vaquero et al 2005b, Wang et al 2006, Ziemons et al 2005), continuous scintillator crystals in conjunction with avalanche photodiode (APD) arrays (Joung et al 2002) or position-sensitive photomultipliers (Benlloch et al 2007), as well as non-crystal-based detector systems, such as multiwire proportional chambers (Schafers et al 2005, Jeavons et al 1999) or semiconductor materials (Ishii et al 2007).

Small animal PET studies generally require spatial resolution capabilities in the range of $1 \mathrm{~mm}$ or less. In PET systems based on arrays of pixelated crystals, the requirements of high resolution and sensitivity can be satisfied by using detector modules composed of narrow and long crystals, and placed in a gantry with the smallest possible diameter (distance between opposite modules). However, long pixelated crystals lead to significant depth of interaction (DOI) detection uncertainties, resulting in a radial elongation artefact that progressively degrades the spatial resolution as the source location is moved away from the centre of the field of view (FOV). Short pixelated crystals reduce the DOI effect however at the same time lead to a reduced detection efficiency. Detector modules with DOI measurement capability have been proposed to simultaneously achieve high resolution and sensitivity (McElroy et al 2005, Tsuda et al 2006, Herrero et al 2007), nevertheless several PET devices for animal imaging employ detectors that do not provide DOI information. In such cases, accurate modelling of the crystal response within the image reconstruction algorithm becomes critical in order to achieve high spatial resolution. The increase of the gantry's diameter can reduce the DOI effect however at the same time leads to a reduction of sensitivity and a degradation of the spatial resolution due to non-colinearity of $\gamma$-ray pairs.

Statistics provides a versatile framework to study the inverse problem of image reconstruction from projections, i.e. guessing unknown data from indirect observations is treated as the problem of inferring properties of an unknown distribution. Iterative statistical 3D reconstruction algorithms, such as the maximum-likelihood expectation-maximization (MLEM) algorithm (Shepp and Vardi 1982), have shown superior image quality and resolution/noise trade-off than classical analytic reconstruction techniques (Chatziioannou et al 2000, Jacobson et al 2000, Johnson et al 1997, Mesina et al 2003) such as filtered backprojection (FBP) (Colsher 1980) and 3D reprojection (3DRP) (Kinahan and Rogers 1989). Statistical methods have the advantage of allowing the incorporation of accurate models of the data acquisition process within the image reconstruction process. In addition, these algorithms can easily overcome artefacts caused by detector gaps or crystal failures as they can efficiently handle missing data in the projection datasets (Ortuño et al 2003, 
Weber et al 2006). Furthermore, the MLEM demonstrates some interesting properties, such as conservation of the total number of acquired counts and nonnegativity of the resulting images.

The main disadvantage of iterative 3D image reconstruction is its high computational cost, which has been partially solved with the development of fast algorithms, such as the ordered-subset, expectation-maximization algorithm (OSEM) (Hudson and Larkin 1994) or multiresolution techniques (Ranganath et al 1988, Raheja et al 1999). In the OSEM algorithm, the image is more often updated, by employing subsets of LORs in the projection and backprojection steps, which has been shown to reduce the time to achieve an image equivalent to that obtained using MLEM by a factor proportional to the number of subsets employed. Rebinning methods such as single slice rebinning (SSRB) (Daube-Witherspoon and Muehllehner 1987) or Fourier rebinning (FORE) (Defrise et al 1997) can reduce the dimensionality of $3 \mathrm{D}$ acquisitions by performing a set of $2 \mathrm{D}$ reconstructions to obtain volumetric data, although there is a loss of image quality with respect to the more timeconsuming fully 3D implementation.

Iterative image reconstruction in PET is an ill-conditioned inverse problem where the noise level can be much higher in the result than in the measured data (Leahy and Qi 2000). The ill-posedness of maximum likelihood leads to an increase of the noise level as iterations proceed. As a result, the iterative procedure has to be stopped early enough in order to guarantee an acceptable resolution/noise trade-off. This inherent property of iterative MLbased PET image reconstruction can be overcome with some sort of regularization techniques which control the noise propagation across iterations.

Many regularization techniques have been proposed, such as Bayesian maximum $a$ posteriori (MAP) formulations (Green 1990, Qi et al 1998) which apply a penalty function to those voxels whose values differ significantly from their neighbours, under the assumption that in emission tomography the reconstructed image should not contain abrupt edges. Regularization methods manage to control noise amplification reducing at the same time the maximum effective spatial resolution in the obtained images.

Image reconstruction methods require a system response model or 'system matrix' (SM) $A=\left\{a_{j i}\right\}$. In terms of MLEM-based iterative image reconstruction, each SM value represents the probability that an event emitted from the source area covered by a specific voxel $i$ is registered by a specific detection unit $j$, i.e. a detector pair or a sinogram bin.

For practical implementations, the SM can be computed either analytically or by simulation. In the former case, SM values can be roughly approximated by calculating the intersection length of the line-of-response (LOR) that connects the pair of detectors with each voxel (Siddon 1985, Herman and Meyer 1993) or using some interpolation method in the way it is implicitly done by classical analytical methods. If crystal sizes are taken into account, calculation of the area of intersection improves the estimation of detection probability in the 2D case (Terstegge et al 1997). A more accurate approach for 3D reconstruction involves the analytical calculation of the volume of intersection between voxels and tubes-of-response (TOR) (Scheins et al 2006).

Geometrical approximations of the SM can be enhanced by including specific analytical models to compute spatially variant crystal penetration effects. 2D approximations have been proposed (Selivanov et al 2000, Strul et al 2003, Yamaya et al 2005). In 3D methods, the complexity of exact calculations (de la Prieta et al 2006) has suggested the use of multiple ray-tracing methods to approximate volumes of intersection and physical properties of crystals (Moehrs et al 2008, Yamaya et al 2008). 
The SM can also be derived from measurements based on point-source acquisitions (Frese et al 2003, Panin et al 2006, Tohme and Qi 2009) or simulations (Alessio et al 2006). The system response function is used in that case to model the spatially variant component of the SM.

Other popular methodology for the estimation of the SM values is the modelling of the system response by Monte Carlo (MC) simulations, allowing the incorporation to the model of the most relevant physical effects involved. MC simulations for SM calculations have yielded results equally good or even better than analytical approaches for SPECT image reconstruction (Lazaro et al 2005). Also fully 3D PET image reconstruction using MC-based SM is feasible for high-resolution PET: general MC codes for high-energy particles' simulation have been used (Rafecas et al 2004), MC codes oriented to PET modelling (Gimenez et al 2006, Rannou and Chatziioannou 2004, Shokouhi et al 2004) or MC codes specifically designed for the SM generation (Herráiz et al 2006).

In either case (measured SM or estimated by MC techniques or analytical techniques) the SM values are pre-calculated offline and stored. The precalculated SM is a valid approach for a specific device, except for the object-dependent effects, basically scatter and attenuation in the source.

Alternatively, the SM can be factorized as a product of independent contributions: geometry, attenuation, positron range, detector blurring and detector sensitivity (Qi et al 1998). This scheme can drastically reduce the size of the SM stored, however introduces at the same time over-simplifications since the SM cannot be expressed in the general case as a product of diagonal matrices.

The SM for 3D reconstruction methods for high-resolution small animal PET scanners contain elements of the order of trillions (both the number of voxels and the number of LORs are typically above the million). This imposes important computing demands in terms of the time required to complete the iterative reconstruction procedure and the computer memory needed for the storage of the SM in case of using a precalculated one.

The system matrices of most PET devices demonstrate a high degree of sparsity, i.e. the vast majority of the SM elements are nearly zero (Kontaxakis et al 2002). This important SM feature is related to the colinearity of each pair of $\gamma$-rays detected and moderate scattering in the object. Thus, if scatter in object is neglected, only voxels intersected by the LOR between the two detectors in coincidence have non-zero probabilities in the column of the SM that corresponds to this detection unit. Modelling of object scatter in the SM reduces drastically the sparseness, resulting into a prohibitive reconstruction time. On the other hand, simplified analytical models of the SM trade accuracy for speed obtaining an excessively sparse SM. In order to do this, physical processes such as the effect of positron range, non-colinearity of the emitted gammas or scatter in crystal are neglected. The use of sparse matrix techniques and system symmetries (Johnson et al 1995) when storing and using the SM elements represents an efficient solution to the problem of the high associated computational cost. In addition, SM pre-calculation can be a very time consuming process even when it needs to be performed only once for a specific scanner configuration.

We have developed and validated a new OSEM-based fast image reconstruction methodology for high-resolution small animal PET scanners based on planar rotating detectors composed of pixelated crystals. The system rotates in a continuous manner and both $3 \mathrm{D}$ and $2 \mathrm{D}$ iterative image reconstruction modes have been developed. In the $3 \mathrm{D}$ case, the difficulties regarding the large size of the SM are overcome with the use of a sparse precalculated SM and axial and transaxial symmetries. 


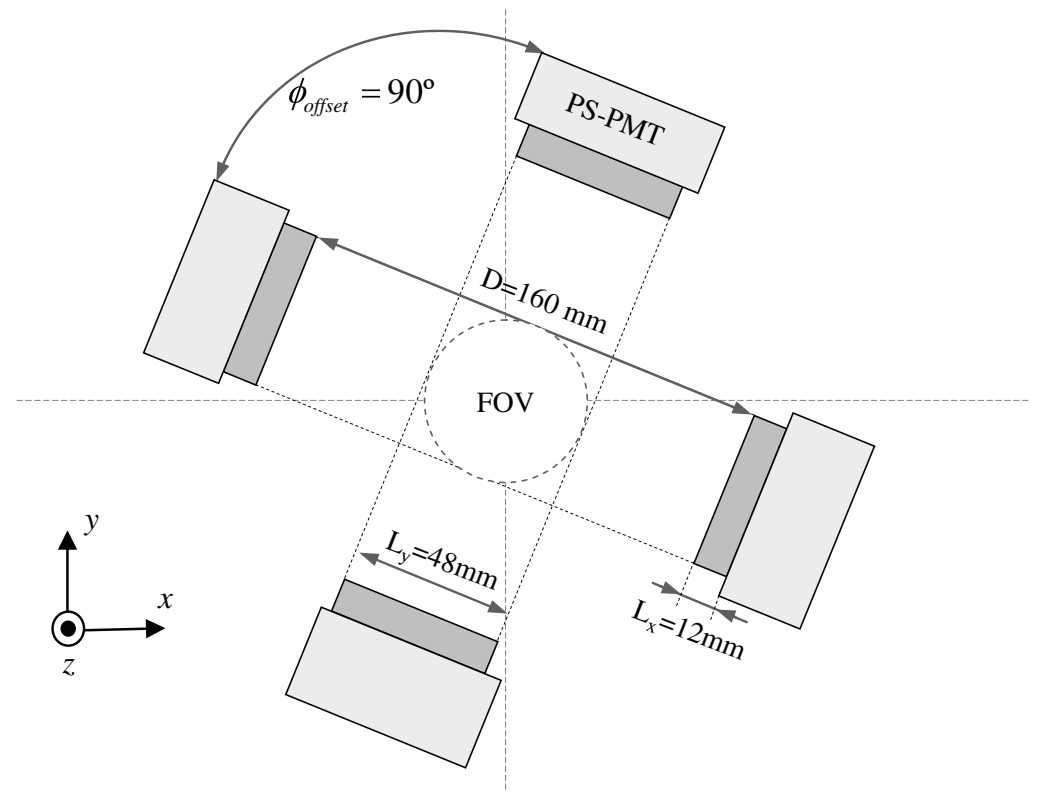

Figure 1. Scheme of the scanner geometry employed in this work. Two pairs of planar detectors composed of pixelated crystals and mounted on a rotating gantry.

\section{Materials and methods}

\subsection{Scanner geometry}

The small animal PET scanner design modelled here consists of two pairs of planar detector modules in coincidence mode, with $160 \mathrm{~mm}$ of separation between opposite modules (Vaquero et al 2004). Each detector is composed of a $30 \times 30$ array of $1.5 \mathrm{~mm} \times 1.5 \mathrm{~mm} \times$ $12.0 \mathrm{~mm}$ LSO pixelated crystals, assembled on a $100 \mu \mathrm{m}$ thick matrix of a plastic reflector. The detectors are mounted on a gantry rotating in continuous mode with $180^{\circ}$ span. A schematic of the device's configuration is shown in figure 1.

The rotating scheme selection was motivated to allow the integration of a coplanar x-ray tomograph in the same gantry (Vaquero et al 2005a, Lage et al 2009) as well as to reduce the number of pairs of detectors in coincidence in order to also reduce the cost of the scanner. In the flat head based scanner proposed, parallax error due to DOI has low values in the extremes of the FOV because LORs involve parallel crystals. Meanwhile, parallax error in the centre of the FOV is also affected by non-parallel crystals (figure 2).

The coincidences associated with pixelated crystals located in the detector extremes are not used in the reconstruction process, and the dimensions of the useful FOV with complete sinograms are $44.8 \mathrm{~mm} \times 44.8 \mathrm{~mm} \times 44.8 \mathrm{~mm}$. Discarding the pixelated crystals at the borders of each detector is based on the assumption that real mounted systems hardly resolve the frontier pixelated scintillators (Vaquero et al 2005b).

Coincidences collected in list mode are binned in direct and oblique sinograms (Defrise et al 1997) as a function of four variables $\{s, \phi, \theta, \Delta\}$, where $s$ is the distance between the $z$ axis and the projection of the LOR onto a transaxial plane; $\phi$ is the LOR azimuthal angle; $\theta$ 


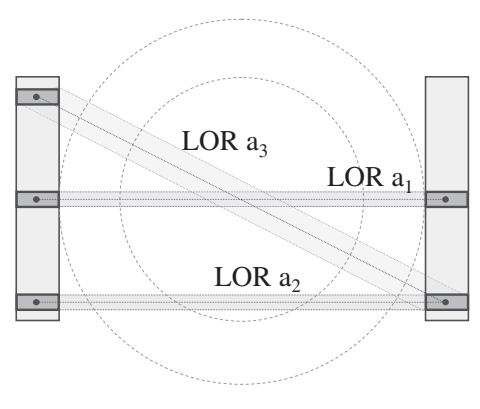

Planar detectors

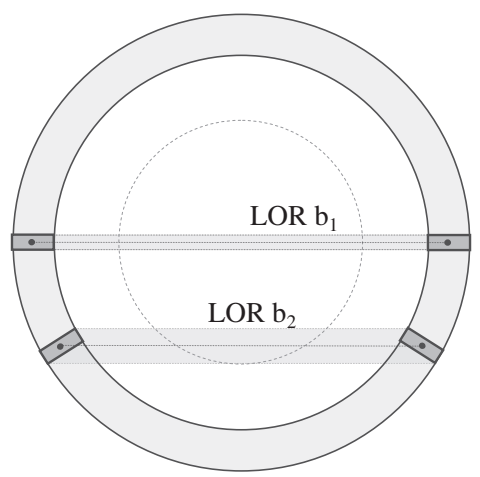

Ring scanner

Figure 2. Sketch of the parallax error in a flat head scanner compared with a full ring scanner.

is the polar angle between the LOR and the transaxial plane and $\Delta$ is the $z$ coordinate of the LOR at its mid-point.

In the proposed rotating scanner geometry, the presence of unnormalizable zero efficiency sinogram bins is avoided by employing a sampling scheme of $0.8 \mathrm{~mm}$ in $s$ and $\Delta$. A sampling distance of $1.5^{\circ}$ in $\phi$ is chosen, which allows us to use a wide range of subsets with a maximum loss of axial resolution of $0.6 \mathrm{~mm}$ in the extreme of the axial FOV, resulting in a total projection size of $55 \times 120 \times 28 \times 28=5174400$ sinogram bins.

\subsection{Reconstruction algorithm}

The regularized OSEM algorithm follows the next iterative scheme:

$$
\begin{aligned}
& \lambda_{i}^{(n, m+1)}=d_{i}^{(n, m)} c_{i}^{(n, m)} \lambda_{i}^{(n, m)}, \quad m=0, \ldots, M-2 \\
& \lambda_{i}^{(n+1,0)}=d_{i}^{(n, M-1)} c_{i}^{(n, M-1)} \lambda_{i}^{(n, M-1)} .
\end{aligned}
$$

During each OSEM sub-iteration $m$, every voxel $i$ of the image $\lambda$ is updated by a multiplicative term $c_{i}$, and, in the MAP scheme applied in this work, regularized by $d_{i}$ (Kontaxakis et al 2002). $\lambda_{i}^{(n, m)}$ is the estimate of the activity concentration in voxel $i$ at iteration $n$ and OSEM sub-iteration $m, M$ being the number of subsets; $c_{i}$ contains the forward and back-projection:

$$
c_{i}^{(n, m)}=\frac{1}{s_{i}^{(m)}} \sum_{j \in \mathbf{s}(m)} \frac{\rho_{j} a_{j i}}{\sum_{i^{\prime}} a_{j i^{\prime}}, \lambda_{i^{\prime}}^{(n, m)}}
$$

where $a_{j i}$ are the SM elements associated with voxels $i$ and detection units $j ; \rho_{j}$ denotes the number of events recorded by each detection unit. The summatory in $j$ runs over the set of detection units in each subset $\mathbf{s}(m)$. The term $s_{i}^{(m)}$ represents the sensitivity image (with respect to one single subset), i.e. the cumulative probability that an event emitted from voxel $i$ is detected by the device if only the LORs assigned to the current subset are counted:

$$
s_{i}^{(m)}=\sum_{j \in \mathbf{s}(m)} a_{j i} .
$$

Finally, the regularization term $d_{i}^{(n, m)}$ has the following expression:

$$
d_{i}^{(n, m)}=\left(1+\beta_{r} \frac{\lambda_{i}^{(n, m)}-f\left(\lambda_{i}^{(n, m)}\right)}{f\left(\lambda_{i}^{(n, m)}\right)}\right)^{-1}
$$


where $\beta_{r}$ is the weight of the prior, constrained to $0<\beta_{r} \leqslant 1 . f(\cdot)$ is a filter that can be of median type (Alenius et al 1998), i.e. estimated as the median of the pixels in a neighbourhood

$\lambda_{i}^{(n, m)}$, generalized median (Alenius and Ruotsalainen 2002), as well as Gaussian type.

In this work, no pre-corrections for random events, attenuation or scatter have been considered for the acquired data, in order to maintain the Poisson statistics as required by MLEM and OSEM algorithms (Fessler and Hero 1995). Such corrections can be incorporated into the reconstruction procedure itself with appropriate modelling, although such effects have not been considered in this work.

\section{3. $3 D$ system matrix modelling}

SM values are computed once offline and stored on disk before the beginning of the reconstruction process in a sparse matrix format, taking advantage of system symmetries that allow us to model and store only a small part of the total $a_{j i}$ elements. In this work we propose MC simulation to model the precalculated SM.

The Geant4 Application for Tomographic Emission (GATE) software package (Jan et al 2004) is quite popular for PET and SPECT simulations and is well validated for a wide range of cameras. However, the complete tracking of high-energy events from the source to the detectors leads to excessively long computation times when high statistics are required for thousands of SM elements. Other MC specific codes for PET simulation (España et al 2009, Lewellen et al 1998) exhibit the same behaviour. The need of a relatively simple but at the same time faster simulation has led to the implementation and validation of a custom MC code for SM modelling.

The custom SM simulation code is based on the generation of a high number of events for every voxel located in the FOV, excluding those voxels for which SM coefficients can be calculated using symmetries. Annihilations are simulated uniformly over the voxel. Each simulated voxel models a SM column, or equivalently, a projection set, that is represented as a set of plane and oblique sinograms.

The Positron range effect and non-colinearity of $\gamma$-rays for a specific radionuclide, i.e. ${ }^{18} \mathrm{~F}$, depend on the material that constitutes the object and it is reasonable to work with an $a$ priori value for water, which approximates the majority of biological tissues. The positron range is modelled with the sum of two exponentials (Levin and Hoffman 1999) which are simulated in the MC code with an accept-reject algorithm. On the other hand, non-colinearity of $\gamma$-rays has been modelled as a Gaussian distribution with $\sigma=0.212^{\circ}$ (de Benedetti et al 1950).

A pair of gamma rays is generated uniformly only in directions within the solid angle of coincidence, which is relatively small in the proposed scanner geometry. Detectors' rotation is modelled with random and uniformly distributed complementary rotation of sources.

Variance reduction (VR) techniques are employed in order to get a larger rate of simulated equivalent events for better statistics in the generated data. This technique is applied to the gamma ray tracking in the scintillator crystal with an auxiliary look-up table (LUT) where relative probabilities of detection in neighbouring crystals have been stored, according to the angle and relative point of intersection with the detector. The LUT sampling interval is $2^{\circ}$ in the angular direction (both in $\theta$ and $\phi$ angles in spherical coordinates, relative to the detector surface), and $1 / 15$ th of the crystal dimensions. Using this technique, complete simulation for every $\gamma$-ray that reaches the detector surface is replaced by pre-calculated detection probabilities that have been modelled as a function of the angle of incidence and intersection point. 
The detector response model is performed with a MC simulation which includes photoelectric and Compton scatter interactions of $\gamma$-rays, which have been modelled according to the negative exponential attenuation law and the Klein-Nishina distribution sampled with a double-rejection technique (Hua 1997). NIST (National Institutes of Standards and Technology) photon cross section tables for data have also been used (Berger et al 1998).

A sufficient number of $\gamma$-rays are launched for every sampled direction of the LUT. The resulting density distribution of detected energy is further integrated over the pixelated crystal volumes. To reduce the size of the stored LUT and reject negligible probabilities, only the $N$ most probable pixelated crystals associated with each incoming angular direction are stored in the LUT. A detailed description of the modelling of the detector interaction can be found in our previous works (Ortuño et al 2006, 2004).

Optionally, the VR method can be skipped, i.e MC simulation of the detector is performed for every $\gamma$-ray that reaches the detector. In the case of multiple interactions, the LOR is positioned according to the centre of the pixelated crystal with maximum deposited energy. If the total energy is lower than the energy window, the event is discarded. The significant increase of simulation time and size of SM due to resulting non-zero elements using this method did not recommended it for fast 3D reconstructions.

This simulation software has been adapted and optimized here for the PET scanner geometry under study. Simulation results are directly processed and stored in sparse-mode format. Finally, after simulation is completed, data are post-processed to distribute $a_{j i}$ values among the chosen number of subsets.

In MC methods, the statistical quality of the SM is determined by the number of simulated events and the VR techniques used to accelerate the modelling process. It is thus desirable to simulate as many coincidences as possible, but it is difficult to know a priori if the simulated data will produce an accurate enough description of the system response. The accuracy of the SM can be estimated with the 'mean relative error' (Rafecas et al 2004) denoted as $\bar{\sigma}_{\text {rel }}$, which is calculated according to (5):

$$
\bar{\sigma}_{\mathrm{rel}} \equiv \frac{1}{N_{\mathrm{nz}}} \sum_{i, j} \frac{\sigma\left(a_{j i}\right)}{a_{j i}}
$$

where $N_{\mathrm{nz}}$ is the number of the non-zero SM elements and $\sigma\left(a_{j i}\right)$ is the standard deviation of their values $a_{j i}$, assuming the Poisson noise: $\sigma\left(a_{j i}\right)=\sqrt{a_{j i}}$. The motivation for using the mean relative error is the following. Ideally, increasing the simulation time implies adding events to elements $a_{j i}$, reducing their variance, while $N_{\mathrm{nz}}$ would remain nearly constant when all SM elements with non-negligible probabilities in the assumed model have been reached. The consequence is that $\bar{\sigma}_{\text {rel }}$ decreases rapidly as the number of simulated events increases, until a reasonably good description of the scanner is reached, beyond which the decrease of $\bar{\sigma}_{\text {rel }}$ is more gradual. Instead of calculating $\bar{\sigma}_{\text {rel }}$ as in (5), something that is only possible when the calculation of the whole SM is completed, a partial value of $\bar{\sigma}_{\text {rel }}$ associated with single voxels $i_{0}$ has been studied (6):

$$
\bar{\sigma}_{\text {rel }}\left(i_{0}\right) \equiv \frac{1}{N_{\mathrm{nz}, i=i_{0}}} \sum_{i=i_{0}, j} \frac{\sigma\left(a_{j i}\right)}{a_{j i}} .
$$

\subsection{System matrix axial symmetries}

A SM symmetry relation can be expressed as $a_{j i}=a_{j^{\prime} i^{\prime}}$ for any voxels $i$ and $i^{\prime}$, and projection bins $j$ and $j^{\prime}$. Parallel shift redundancies and reflection axial symmetry (Johnson et al 
Z-parallel translation

Z-reflection symmetry

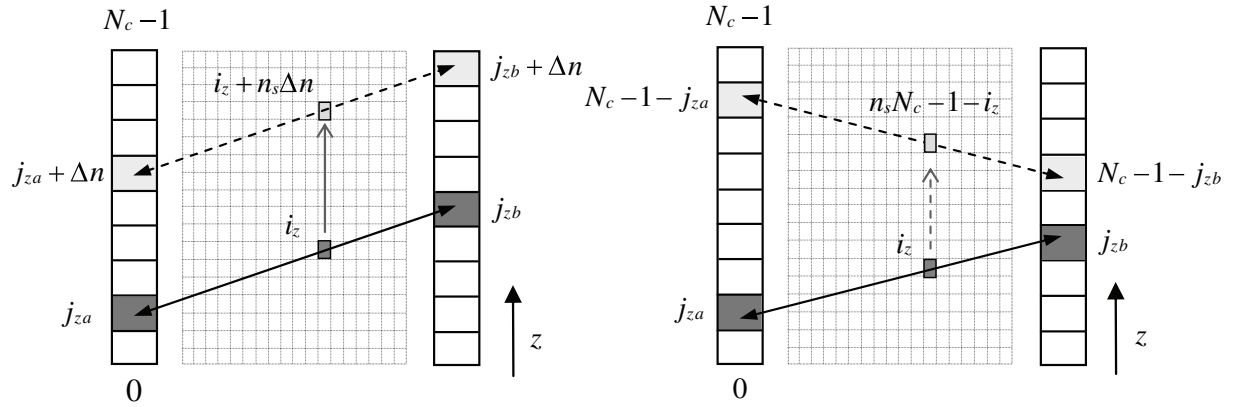

Figure 3. Schematic of parallel shift (left) and axial symmetry (right) along the axial direction

1995) can reduce the storage size of the SM by a factor proportional to the number of transaxial slices. Both are applicable in scanners with translation symmetry along the axial axis (figure 3).

Voxels were chosen in an orthogonal grid oriented along the $x, y$ and $z$ axes, with $z$ being the axial axis of the PET device. Choosing the voxel size in $z$ as a divisor of the pixelated crystal size in the $z$ direction, translation symmetry can be applied. Analogously, reflection axial symmetry needs an alignment between the orthogonal grid and the pixelated crystals. If the orthogonal grid is employed as a discretization of the volume vector $\lambda$, each voxel $i$ will have Cartesian coordinates $[x, y, z]$ directly related to voxel indices in the $x, y$ and $z$ axes, denoted as $\left\{i_{x}, i_{y}, i_{z}\right\}$. Sampled $z$ values as a function of the voxel size $\Delta z_{v}$ are

$$
z_{v}=\left(i_{z} \Delta z_{v}, i_{z}: 0, \ldots, N_{z}-1\right)
$$

where $N_{z}$ is the number of $z$ axis voxel indices.

On the other hand, the projection index $j$ is assigned to one LOR joining two pixelated crystals. Scanners consisting of pairs of opposed detectors at the same distance can assign the LOR directly to a discrete index depending on the pixelated crystal row:

$$
\begin{aligned}
& z_{a}=\left(j_{z a} \Delta z_{c}, j_{z a}: 0, \ldots, N_{c}-1\right) \\
& z_{b}=\left(j_{z b} \Delta z_{c}, j_{z b}: 0, \ldots, N_{c}-1\right)
\end{aligned}
$$

where $\Delta z_{c}$ is the crystal thickness in the $z$ axis direction and $N_{c}$ is the number of crystal rows in each detector. $j_{z a}$ and $j_{z b}$ are the LOR indices associated with each crystal row. Using direct and oblique sinograms, these indices are extracted from the decomposition

$$
j=j_{s}+N_{s} j_{\phi}+N_{s} N_{\phi} j_{z a}+N_{s} N_{\phi} N_{z} j_{z b}
$$

where $\left\{N_{s}, N_{\phi}, N_{z}\right\}$ are the number of bins in the $\{s, \phi, z\}$ projection dimensions, $j_{s}$ is the projection bin index in the radial dimension and $j_{\phi}$ is the projection bin index in the angular dimension.

Subset division of the projection space for OSEM algorithm (1) is performed over the $\phi$ dimension in the following way:

$$
j \in \mathbf{s}(m) \Leftrightarrow\left(j_{\phi} \mathrm{MOD} M\right)=m .
$$

$N_{\phi}$ must be divisible by $M$ to have subsets with the same number of elements.

According to the notation presented before, parallel shift redundancies can be applied to get symmetries between parallel LORs from one voxel $i$ with $z$ index $i_{z}$ to a new voxel $i^{\prime}$ with index $i_{z}+n_{s} \Delta n$, where $\Delta n$ is the axial shift expressed in numbers of pixelated crystal 


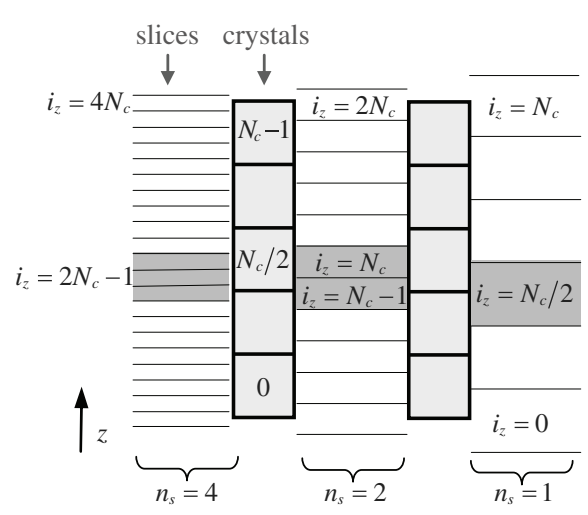

(a)

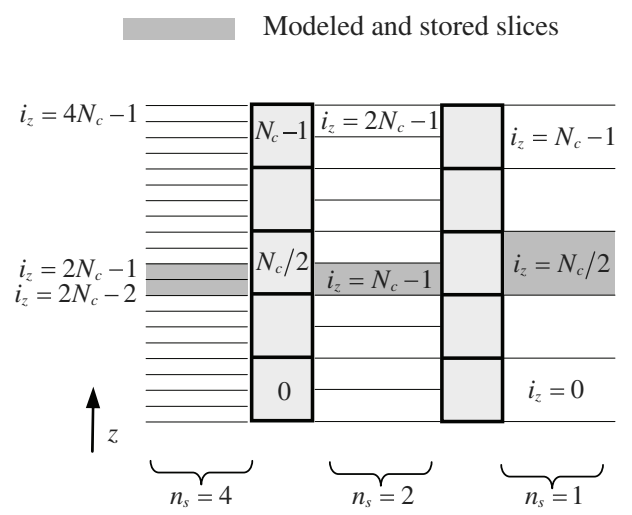

(b)

Figure 4. The number of modelled transaxial slices depends on the thickness and alignment of the voxel grid. With the axial alignment plotted in (b), only two central slices need to be modelled in detail for axial voxel size one-quarter the pixelated crystal width, and only one slice for thicker voxels. In alignment (a) at least three slices are modelled in detail to obtain the SM values for the rest of the slices with axial symmetries and translations. Configuration (a) is denoted as centred alignment and configuration (b) is denoted as shifted alignment.

rows, and $n_{s}$ denotes the number of slices that match the pixelated crystal thickness in the $z$ dimension, $n_{s}=\Delta z_{c} / \Delta z_{v}$. The index $j^{\prime}$ of the LOR symmetric to the LOR with the index $j$ involves the axial shift $\Delta n$ between the two pairs of crystal rows:

$$
a_{j i}=a_{j^{\prime} i^{\prime}} \Leftrightarrow\left\{\begin{array}{l}
i_{z}{ }^{\prime}=i_{z}+n_{s} \Delta n \\
j_{z a}^{\prime}=j_{z a}+\Delta n \\
j_{z b}^{\prime}=j_{z b}+\Delta n
\end{array} .\right.
$$

Analogously, the reflection symmetry relation along the axial direction between mirrored LORs with respect to the central transaxial plane can be expressed as follows:

$$
a_{j i}=a_{j^{\prime} i^{\prime}}\left\{\begin{array}{l}
i_{z}^{\prime}=n_{s} N_{z}-i_{z} \\
j_{z a}^{\prime}=N_{z}-1-j_{z a} \\
j_{z b}^{\prime}=N_{z}-1-j_{z b}
\end{array}\right.
$$

The voxel size is chosen at one-quarter of the size of the detector pixelated crystal $\left(n_{s}=4\right)$, to ensure that the intrinsic scanner resolution is maintained both in the transaxial and the axial directions. Coarse grid SM at half the pixelated crystal size has also been modelled to be used when faster reconstructions are desired.

With the axial alignment proposed in figure 4(b), where slice separation coincides with a crystal separation, only two central slices need to be modelled in detail for the finest resolution. This resolution corresponds to a slice thickness of one-fourth of the pixelated crystal size in the axial dimension $\left(n_{s}=4\right)$, while the rest of the slices are obtained with reflection axial symmetry and parallel shift axial redundancies. This slice position will be referred to as shifted alignment for short.

An alternative approach proposed consists of centring one slice with respect to the pixelated crystal centre, as in figure 4(a). This grid alignment in the axial direction, referred to as centred alignment for short, requires the simulation of at least three slices in the finest resolution, resulting in an increase of the simulation time and storage requirements for the 


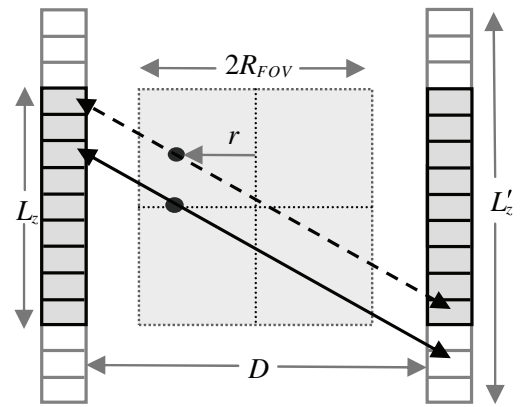

Figure 5. Not all LORs have a symmetry relationship with another one simulated in a central slice, unless the detector is considered longer than it actually is, with the inclusion of virtual crystals.

precalculated SM. Another drawback of the centred alignment is that SM values associated with coarse resolution voxels cannot be extracted with a combination of the values associated with the finest resolution because there is no direct overlap as it occurs in the shifted alignment.

According to the symmetries defined above, the maximum polar angle for a given LOR to match with another one belonging to the central transaxial plane depends on the radial distance $r$ of the event generation point for this LOR, the distance between opposite detectors $D$ and the axial size of detectors $L_{z}$ (figure 5):

$$
\theta_{\max }=\arctan \left(\frac{L_{z}}{D+2 r}\right)
$$

In order to cover the complete set of $3 \mathrm{D}$ acquisitions, with $\theta_{\max }=\arctan \left(L_{z} / D\right)$, the SM modelling requires the consideration of a virtual extension of the detector in the axial direction $L_{z}^{\prime}$, as shown in figure 5. In terms of the real dimension $L_{z}$, the virtual detector length in $z$ is

$$
L_{z}{ }^{\prime}=L_{z}(D+2 r) / D \text {. }
$$

In summary, axial symmetries allow for calculating all SM values by performing complete MC-based calculations of the SM values that correspond to the voxels of a limited number of central slices. This number varies from 1 to 3 depending on the slice alignment and slice thickness. One requirement is that the polar angle $\theta$ be limited; otherwise, the extension of the detector with virtual crystals should be considered.

\subsection{System matrix transaxial symmetries}

Since the majority of scanners have rotational symmetry along the axial axis, in-plane transaxial symmetries can also be used in the SM modelling and reconstruction scheme, by modelling only the voxels belonging to the first quadrant, i.e. $i_{x} \in\left[0, N_{x} / 2\right)$, and $i_{y} \in\left[0, N_{y} / 2\right)$. One voxel $i_{a}$ has an in-plane rotational symmetry relationship with voxels $i_{b}, i_{c}$ and $i_{d}$ belonging to the other three quadrants and relative indices according to (15):

$$
\begin{aligned}
i_{a} & =\left\{i_{x}, i_{y}\right\} \\
i_{b} & =\left\{N_{x}-i_{x}-1, N_{x}-i_{y}-1\right\} \\
i_{c} & =\left\{i_{y}, N_{x}-i_{x}-1\right\} \\
i_{d} & =\left\{N_{x}-i_{y}-1, i_{x}\right\} .
\end{aligned}
$$

Each of the above cases involves a mapping of the azimuthal angle $\phi$ of the LOR: $180^{\circ}$ $\left(i_{a} \rightarrow i_{b}\right), 90^{\circ}\left(\left(i_{a} \rightarrow i_{d}\right)\right.$ and $-90^{\circ}\left(i_{a} \rightarrow i_{c}\right)$. The changes of sinogram and image indices 
Table 1. SM rotation symmetries in the transaxial plane.

\begin{tabular}{llll}
\hline$i_{a} \rightarrow i_{b}$ & $i_{a} \rightarrow i_{c}$ & $i_{a} \rightarrow i_{d}$ & $\left(\forall j_{\phi}\right)$ \\
$\phi \rightarrow(\phi+\pi)$ & $\phi \rightarrow(\phi-\pi / 2)$ & $\phi \rightarrow(\phi+\pi / 2)$ & \\
\hline$i_{x}{ }^{\prime}=N_{x}-i_{x}-1$ & $i_{x}{ }^{\prime}=i_{y}$ & $i_{x}{ }^{\prime}=N_{x}-i_{x}-1$ & $\left(\forall j_{\phi}\right)$ \\
$i_{y}{ }^{\prime}=N_{x}-i_{y}-1$ & $i_{y}{ }^{\prime}=N_{x}-i_{x}-1$ & $i_{y}{ }^{\prime}=i_{x}$ & \\
$j_{z a}^{\prime}=j_{z b}$ & $j_{z a}^{\prime}=j_{z b}$ & $j_{z a}^{\prime}=j_{z a}$ & $\left(\right.$ if $\left.j_{\phi}<N_{\phi} / 2\right)$ \\
$j_{z b}^{\prime}=j_{z a}$ & $j_{z b}^{\prime}=j_{z a}$ & $j_{z b}^{\prime}=j_{z b}$ & \\
$j_{s}^{\prime}=N_{s}-j_{s}-1$ & $j_{s}^{\prime}=N_{s}-j_{s}-1$ & $j_{s}^{\prime}=j_{s}$ & \\
$j_{\phi}^{\prime}=j_{\phi}$ & $j_{\phi}^{\prime}=j_{\phi}+N_{\phi} / 2$ & $j_{\phi}^{\prime}=j_{\phi}+N_{\phi} / 2$ & \\
$j_{z a}^{\prime}=j_{z b}$ & $j_{z a}^{\prime}=j_{z a}$ & $j_{z a}^{\prime}=j_{z b}$ & $\left(\right.$ if $\left.j_{\phi} \geqslant N_{\phi} / 2\right)$ \\
$j_{z b}^{\prime}=j_{z a}$ & $j_{z b}^{\prime}=j_{z b}$ & $j_{z b}^{\prime}=j_{z a}$ & \\
$j_{s}^{\prime}=N_{s}-j_{s}-1$ & $j_{s}^{\prime}=j_{s}$ & $j_{s}^{\prime}=N_{s}-j_{s}-1$ & \\
$j_{\phi}^{\prime}=j_{\phi}$ & $j_{\phi}^{\prime}=j_{\phi}-N_{\phi} / 2$ & $j_{\phi}^{\prime}=j_{\phi}-N_{\phi} / 2$ & \\
\hline
\end{tabular}

are shown in table 1. According to (10), these symmetries link projection bins contained in the same subset. It should be noted that the implementation of the angular shifts of $90^{\circ}$ and $180^{\circ}$ do not involve any interpolation if the sinogram size in $\phi$ is even.

In addition to the rotation symmetry, transaxial in-plane reflection symmetry has also been applied. In this case, the transpose of $i_{x}$ and $i_{y}$ voxel indices lead to the $\phi$ inversion. Symmetry relations are given as follows:

$$
\begin{aligned}
& a_{j i}=a_{j^{\prime} i^{\prime}} \Leftrightarrow\left\{\begin{array}{l}
i_{x}{ }^{\prime}=i_{y} \\
i_{y}{ }^{\prime}=i_{x} \\
j_{\phi}{ }^{\prime}=N_{\phi} / 2-j_{\phi}-1 \\
j_{s}{ }^{\prime}=j_{s}
\end{array}\right\} \\
& a_{j i}=a_{j^{\prime} i^{\prime}} \Leftrightarrow\left\{\begin{array}{l}
i_{x}{ }^{\prime}=i_{y} \\
i_{y}{ }^{\prime}=i_{x} \\
j_{z a}{ }^{\prime}=j_{z b} \\
j_{z b}{ }^{\prime}=j_{z a} \\
j_{\phi}{ }^{\prime}=3 N_{\phi} / 2-j_{\phi}-1 \\
j_{s}=N_{s}-j_{s}-1
\end{array}\right\} \quad \text { if } j_{\phi}<N_{\phi} / 2 .
\end{aligned}
$$

Note that the transaxial in-plane reflection symmetry connects elements belonging to different OSEM subsets. For the sake of simplicity, this symmetry is precalculated off-line and only the part of the SM that corresponds to one subset is loaded in computer memory during each OSEM sub-iteration.

Figure 6 shows the location of voxels needed to be simulated using both transaxial reflection and rotation symmetries, which are approximately one-eighth part of the total voxels contained in one transaxial slice.

\subsection{System matrix storage}

The calculated values of the SM are stored in sparse mode, and every non-zero $a_{j i}$ element is associated with a position index and the corresponding probability value. Both data are kept in two classes of files that are specifically structured for fast access and further processing 

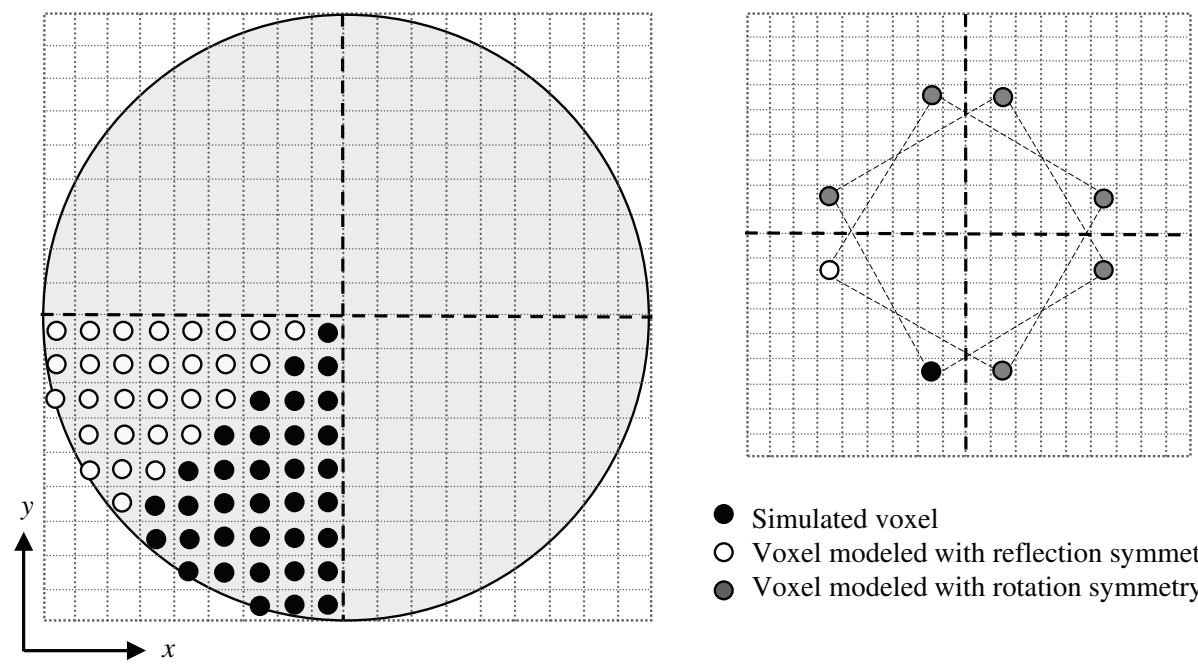

- Simulated voxel

O Voxel modeled with reflection symmetry

○ Voxel modeled with rotation symmetry

Figure 6. Schematic of modelled voxels in the transaxial plane. Only the values of the SM elements that involve the eighth part of voxels belonging to one transaxial slice need to be modelled. The rest of the values in the precalculated SM can be obtained by applying rotational and reflection symmetries.

Table 2. Number of SM elements and number of simulated voxels for some grid discretizations.

\begin{tabular}{llll}
\hline SM type & Voxels $(i)$ & $i \times j$ & Voxels simulated \\
\hline $\mathrm{SM}_{c}$, shifted alignment & $56 \times 56 \times 70$ & $1.77 \times 10^{12}$ & 316 \\
$\mathrm{SM}_{c}$, centred alignment & $56 \times 56 \times 71$ & $1.80 \times 10^{12}$ & 632 \\
$\mathrm{SM}_{b}$, shifted alignment & $112 \times 112 \times 70$ & $7.10 \times 10^{12}$ & 1247 \\
$\mathrm{SM}_{b}$, centred alignment & $112 \times 112 \times 71$ & $7.20 \times 10^{12}$ & 2494 \\
$\mathrm{SM}_{a}$, shifted alignment & $112 \times 112 \times 140$ & $1.42 \times 10^{13}$ & 2494 \\
$\mathrm{SM}_{a}$, centred alignment & $112 \times 112 \times 141$ & $1.43 \times 10^{13}$ & 3741 \\
\hline
\end{tabular}

during the projection and backprojection steps. Stored data are ordered according to the subset subdivision of $j$ to allow sequential access of the required SM elements at each step.

Different discretizations of the image volume have been used in this work, with voxel sizes of $0.4 \mathrm{~mm} \times 0.4 \mathrm{~mm} \times 0.4 \mathrm{~mm}, 0.4 \mathrm{~mm} \times 0.4 \mathrm{~mm} \times 0.8 \mathrm{~mm}$ and $0.8 \mathrm{~mm} \times 0.8 \mathrm{~mm} \times$ $0.8 \mathrm{~mm}$, in the transaxial plane $(x-y)$ and the axial $(z)$ axis. The SM associated with these grids are denoted respectively as $\mathrm{SM}_{a}, \mathrm{SM}_{b}$ and $\mathrm{SM}_{c}$ in short.

The finest grid is intended to guarantee the theoretical limit of spatial resolution in reconstructed PET images using PS-PMT detectors (Lecomte 2004). Coarse voxel sizes are employed to get faster reconstruction times when a regularized image is preferred, rather than a higher resolution but noisier result.

Table 2 shows the exact number of simulated voxels for different grid resolutions and both centred and shifted axial alignments. Symmetries make the repetition of the process for all voxels in the image volume grid unnecessary. However the number of voxels involved in the calculations is still in the order of thousands for the finest resolution of the camera configuration chosen for these experiments. However, the complete set of non-zero $a_{j i}$ 
Table 3. Parameters of modelled SM.

\begin{tabular}{llllll}
\hline SM type & Voxels $(\boldsymbol{i})$ & Disk space $(\mathbf{M B})$ & $\boldsymbol{N}_{\boldsymbol{i j}}$ & Sparsity & $\sigma_{\text {rel }}\left(i_{0}\right)$ \\
\hline $\mathrm{SM}_{c}$, shifted alignment & $56 \times 56 \times 70$ & 95 & 48140 & $99.405 \%$ & 0.23 \\
$\mathrm{SM}_{b}$, shifted alignment & $112 \times 112 \times 70$ & 302 & 37713 & $99.53 \%$ & 0.21 \\
$\mathrm{SM}_{a}$, shifted alignment & $112 \times 112 \times 140$ & 596 & 36408 & $99.54 \%$ & 0.24 \\
\hline
\end{tabular}

elements that correspond to the voxels involved in the Monte Carlo calculations can be loaded in RAM even for the finest resolution.

The total time for the simulations is maintained below $100 \mathrm{~h}$ in a processor Intel Core 2 Duo, with $2.4 \mathrm{GHz}$ CPU clock. This relatively short time of simulation was an important requirement due to the necessity of evaluating different scanner configurations in the design step. For the SM with shifted axial alignments, table 3 shows the disk space, degree of sparsity, $\bar{\sigma}_{\text {rel }}\left(i_{0}\right)$ and mean number of SM elements stored in the disk by pixel (i.e. not equal to zero), denoted as $N_{i j}$.

\section{7. $2 D$ system matrix}

2D image reconstruction can be applied in order to perform fast reconstruction of the 3D acquired data. Data are rebinned in this case with the SSRB or the FORE algorithms. For this case, instead of modelling the 2D SM with an unrealistic MC process in two dimensions, the calculated 3D SM is rebinned in order to obtain the new probability values. For this purpose a procedure based on the SSRB algorithm is employed for all SM entries that correspond to each voxel in the image volume. A maximum axial difference of 15 pixelated crystals is employed in the post-processing of the SM data in this case.

For 2D image reconstruction, in-plane symmetries are not employed since memory requirements do not make necessary this effort. In this case, the probability values associated with one complete transaxial slice are calculated and stored. The same values are employed for the iterative reconstruction of the $2 \mathrm{D}$ rebinned projection data for all transaxial slices.

The SM corresponds to a projection space of $55 \times 120$ bins and an image grid of $112 \times 112$ pixels. The whole volume covered by the FOV is obtained with $N_{z}$ independent $2 \mathrm{D}$ reconstructions; however, regularization filters and anatomical priors implemented are of 3D nature and are applied between sub-iterations. The voxel size is $0.8 \mathrm{~mm}$ in the $z$ direction due to the rebinning process. The precalculated and stored 2D SM has the following characteristics: total number of modelled pixels: 9816; storage size of $24.5 \mathrm{MB}$; total SM elements: 82790 400; mean number of non-zero SM elements per pixel: 513.5, leading to a sparsity of $92.5 \%$ and the mean relative error is 0.11 . In addition to the SM of $112 \times 112$ pixels, a finest grid 2D SM of $224 \times 224$ was also modelled.

\subsection{Experimental validations using synthetic data}

Some synthetic phantoms have been simulated to evaluate and validate the reconstruction method proposed in this work. First, point sources placed in a hot cylinder were simulated with GATE, in order to quantitatively evaluate and also quantify noise and resolution recovery. Secondly, an image quality phantom (NEMA 2008) has also been simulated with GATE. This experiment has been designed to calculate uniformity, recovery coefficients and edge resolution. Thirdly, a Derenzo-type phantom was simulated for qualitative evaluations. 


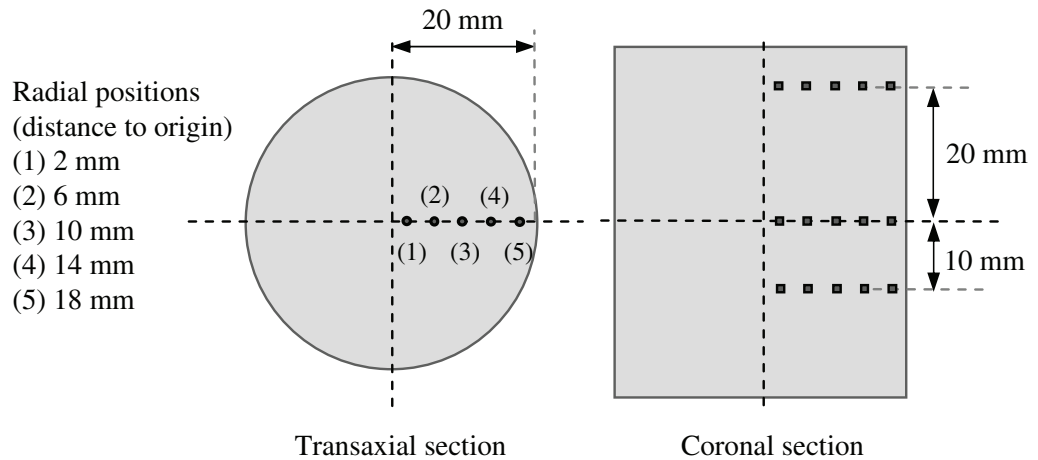

Figure 7. Transaxial and coronal sections of the point-source phantom.

Coincidences from GATE-simulated studies were collected in list-mode format and translated to 3D sinogram format using a binning custom module that does not provide DOI resolution, but calculates the sinogram bin from the centres of the pixelated crystals. The energy window has been fixed to $400-700 \mathrm{KeV}$ and the timing coincidence window to $12 \mathrm{~ns}$. Simulations included the positron range, non-colinearity, scatter and attenuation effects. The radionuclide was ${ }^{18} \mathrm{~F}$.

In the following experiments, 3D and $2 \mathrm{D}$ iterative reconstructions are compared. 3DOSEM reconstruction was performed with two different precalculated SM which differ in the voxel size along the $z$ dimension: $\mathrm{SM}_{a}$ based on an image volume grid of $112 \times 112 \times$ 112 voxels each of size $0.4 \mathrm{~mm} \times 0.4 \mathrm{~mm} \times 0.4 \mathrm{~mm} ; \mathrm{SM}_{b}$ is based on an image volume grid of $112 \times 112 \times 56$ voxels of size $0.4 \mathrm{~mm} \times 0.4 \mathrm{~mm} \times 0.8 \mathrm{~mm}$. Both $\mathrm{SM}$ were modelled with shifted axial alignment. The associated SM applied to the 2D-OSEM reconstruction corresponds in all cases to a $112 \times 112$ D image grid with a pixel size of $0.4 \mathrm{~mm} \times$ $0.4 \mathrm{~mm}$ The reconstruction has been sequentially performed on fifty-five $0.8 \mathrm{~mm}$ thick transaxial planes.

It should be noted that reconstructions employing $\mathrm{SM}_{b}$ for 3D-OSEM produce images with the same voxel size than $2 \mathrm{D}$ methods and require the modelling of only those voxels situated in one transaxial plane, while $\mathrm{SM}_{a}$ corresponds to voxels with half the thickness of those produced by $\mathrm{SM}_{b}$ and requires at least the modelling of two transaxial slices.

2.8.1. Point-source phantom. The phantom, whose transaxial and coronal sections are shown in figure 7 , consists of 15 point sources placed in a warm cylinder with a radius of $20 \mathrm{~mm}$ and length of $48 \mathrm{~mm}$. Radial positions are located at 2, 6, 10, 14 and $18 \mathrm{~mm}$ off-centre in three transaxial planes located at $-20,0$, and $10 \mathrm{~mm}$ from the centre of the FOV. The activity of the cylindrical background was fixed to $12.9 \mu \mathrm{Ci}$, while the activity of each of the point sources was $0.1621 \mu \mathrm{Ci}$ each.

2.8.2. Derenzo-type phantom. A Derenzo-type phantom has been modelled with GATE, as a cylinder of plastic (methacrylate) with $22 \mathrm{~mm}$ radius and sets of active water-filled rods of $4.8,4.0,3.2,2.4,1.6$ and $1.2 \mathrm{~mm}$ in diameter and the same distance between the rod surfaces. Rod lengths have been fixed to $16 \mathrm{~mm}$, and the phantom has been placed in the centre of the FOV. Transaxial and coronal sections of this phantom are shown in figure 8 . Activity density selected was $5 \mu \mathrm{Ci} \mathrm{cm}{ }^{-3}$ leading a total activity of $13.24 \mu \mathrm{Ci}$. 


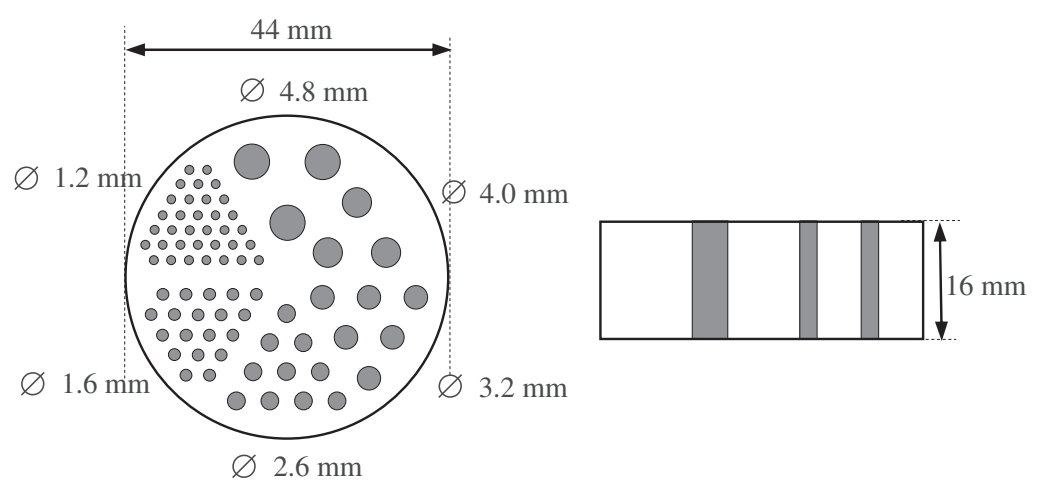

Transaxial section

Coronal section

Figure 8. Derenzo-type phantom with rods of diameters 4.8, 4.0, 3.2, 2.4, 1.6 and $1.2 \mathrm{~mm}$, respectively.

Two acquisitions have been simulated at two different phantom positions. In the first one, the phantom has been placed with rods perpendicular to the scanner axial axis, in order to better evaluate the spatial resolution in the $z$ direction. A total amount of $6.68 \times 10^{6}$ coincidences have been collected for this case. The second alignment has been the usual one: rods parallel to the axial axis, oriented for resolution measurements in the transaxial plane. It should be noted that the axial invariance of rods in this alignment allows obtaining good axial resolution results with rebinning methods. In addition, if $z$ filtering is employed in the regularization scheme, good SNR values can be achieved because of the specific phantom configuration rather than the reconstruction properties. The counts collected with this alignment were $7.26 \times 10^{6}$.

2.8.3. Quality phantom. The image quality phantom with coronal and transaxial sections shown in figure 9 has been designed in order to evaluate the noise characteristics and contrast recovery of the proposed algorithms. The GATE simulation package has been used in this experiment.

The total length of the phantom is $50 \mathrm{~mm}$ although only $44.8 \mathrm{~mm}$ is covered by the FOV of the camera. This phantom consists of two main parts: a main cylindrical chamber with $30 \mathrm{~mm}$ diameter and $30 \mathrm{~mm}$ length, and five fillable rods drilled through solid plastic, with radii of $1,2,3,4$ and $5 \mathrm{~mm}$, and a length of $20 \mathrm{~mm}$ respectively.

The main cylindrical chamber (or background) is filled with water containing ${ }^{18} \mathrm{~F}$ with the same activity concentration of the fillable rods. The cylinder includes two smaller cylinders of $14 \mathrm{~mm}$ length and $8 \mathrm{~mm}$ inner diameter. One of them is filled with air and the other contains water with double activity concentration. Total activity in this phantom was $100 \mu \mathrm{Ci}$.

The per cent contrast recovery for hot ROIs $\left(Q_{H}\right)$ (NEMA 2007) is defined as

$$
Q_{H}=\left(\frac{C_{H} / C_{B}-1}{a_{H} / a_{B}-1}\right) 100(\%)
$$

where $C_{H}$ is the average counts in the hot ROIs, $C_{B}$ is the average counts in the background ROI, and $a_{H}$ and $a_{B}$ are the activity concentrations in the hot ROI and hot background, respectively. If $a_{H}=a_{B}$, an alternative contrast recovery $Q_{H}^{\prime}$ can be calculated as $C_{H} / C_{B}$. 


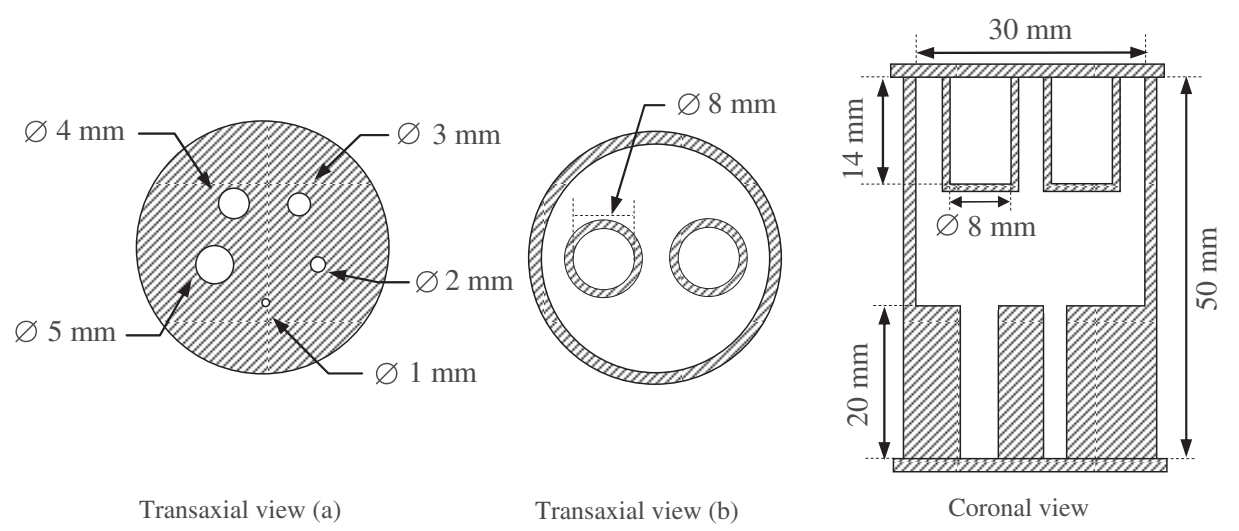

Figure 9. Transaxial and coronal views of the image quality phantom.

On the other hand, contrast recovery for cold ROIs $\left(Q_{C}\right)$ (NEMA 2007) can be measured as

$$
Q_{C}=\left(1-\frac{C_{C}}{C_{B}}\right) 100(\%)
$$

where $C_{C}$ is the average counts in the cold cylinder ROI.

\section{Results}

\subsection{System matrix statistics}

The obtained SM has been compared with results produced by GATE. The comparison has been done with the simulation of two selected voxel projections (i.e. SM columns) because the complete simulation of the SM using GATE was unaffordable due to excessively long computation times.

One voxel was located $15 \mathrm{~mm}$ off the axial axis, in the central transaxial plane, while the other one was considered in the centre of the FOV. In both cases the voxel size was $0.8 \mathrm{~mm} \times$ $0.8 \mathrm{~mm} \times 0.8 \mathrm{~mm}$. GATE simulations were stored in list-mode format and binned to 3D sinogram format with the same module used in the phantom studies.

Each GATE voxel projection simulation was performed until reaching $1 \times 10^{6}$ coincidences, and it took approximately $24 \mathrm{~h}$ to compute in a personal computer. On the other hand, the custom SM simulation needed less than $1 \mathrm{~min}$ to acquire $65 \times 10^{7}$ coincidences by voxel. The speed-up factor is about six orders of magnitude.

SM columns have 5174400 elements, organized as 3D projections of $55 \times 120 \times$ $28 \times 28$ bins. For better appreciation of similarities between GATE and custom simulations, histogrammed results are presented in figure 10 as a matrix of $N_{z} \times N_{z}$ elements where each value is equal to the total coincidences assigned to each $\left\{j_{z a}, j_{z b}\right\}$ oblique sinogram. The grey level values of figure 10 are normalized to the total collected coincidences. The similar distribution of coincidences in oblique sinograms can be appreciated in detail tracing a profile over sinograms with index $j_{z b}=N_{z}-1-j_{z a}$ (figure 10, right).

Figure 11 (left) shows the projection of a voxel located $15 \mathrm{~mm}$ off the axial axis, histogrammed over $\left\{j_{z a}, j_{z b}\right\}$ bin indices, i.e. the sum of the whole set of the oblique sinograms. 
(1) Voxel $15 \mathrm{~mm}$ off the axial axis

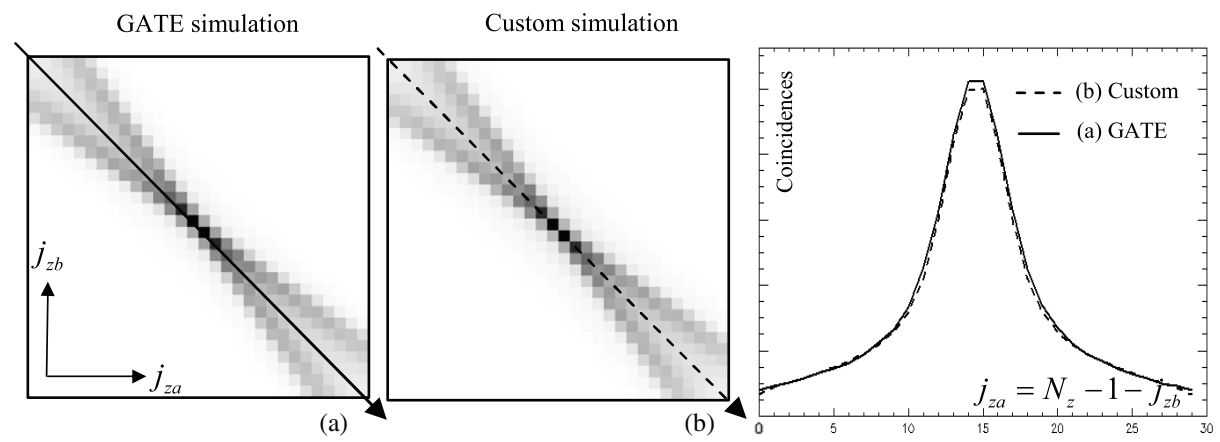

Histograms $h\left(j_{z a}, j_{z b}\right)$

(a) \& (b) profiles

(2) Voxel centered in the axial axis

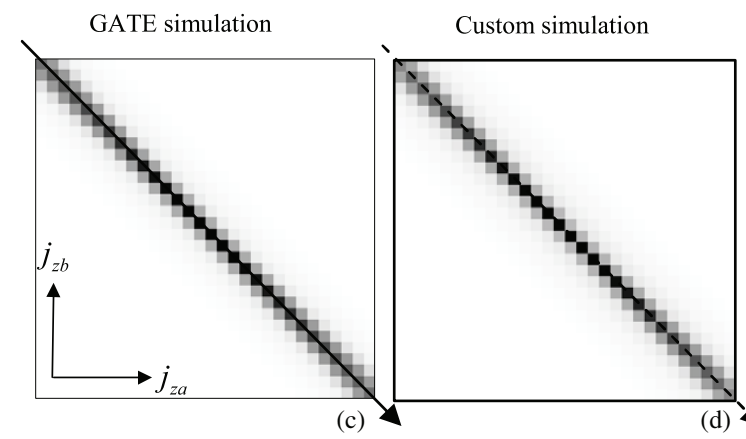

Histograms $h\left(j_{z a}, j_{z b}\right)=\sum_{\forall s, \phi} \rho$

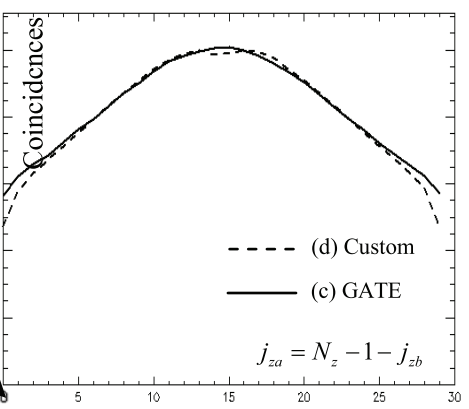

(c) \& (d) profiles

Figure 10. Graphical comparison between voxel simulations with GATE and with the proposed custom model. (1) The voxel was located $15 \mathrm{~mm}$ off the axial axis; (2) voxel centred in the FOV. Histograms with the total value of oblique sinograms represented in grey level, and profiles of the main diagonal are shown.

Single 2D sinograms (figure 11, right) are more suitable for better appreciating the different degree of sparsity between GATE and custom simulations. It can be noted that the VR technique employing the LUT to model crystal interactions limits the non-zero bins due to inter-crystal scatter and produces sinograms with higher degree of sparsity.

The voxel associated with the smallest values of probability of detection, i.e. among the ones located in the extreme of the FOV, and the voxel centred in the FOV, give upper and lower limit values of $\bar{\sigma}_{\text {rel }}$ in the simulation scheme, since the number of events launched per voxel is the same. This effect is observed in figure 12 where the $\bar{\sigma}_{\text {rel }}$ values of extreme and centred voxels of the transaxial central slices are plotted. Also in figure 13, coarse resolutions with large voxels show more non-zero $j$ elements, and for the same SM, the centred voxel has also more non-zero values. The $\bar{\sigma}_{\text {rel }}$ results plotted in figure 12 suggest that launching $2 \times 10^{8}$ events per voxel would lead to reasonably good statistics. 


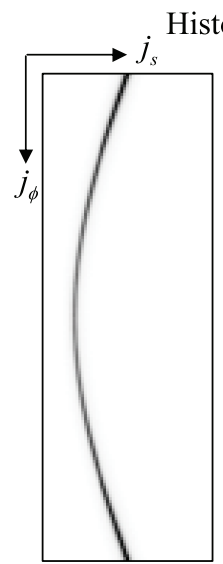

GATE
Histogram $h\left(j_{s}, j_{\phi}\right)=\sum_{\forall z a z b} \rho$

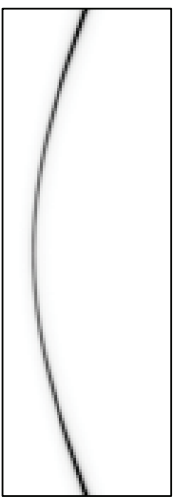

Custom

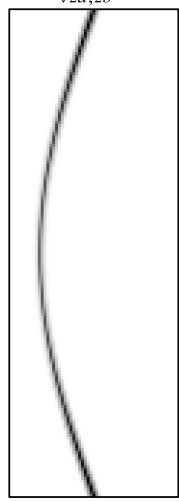

Custom (VR)

Sinogram $\rho_{2 D}\left(j_{s}, j_{\phi}\right) \mid j_{z a}=13, j_{z b}=22$

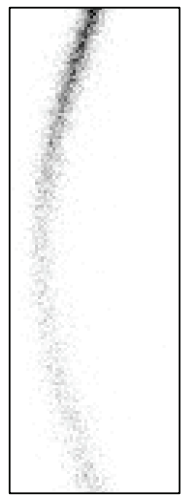

GATE

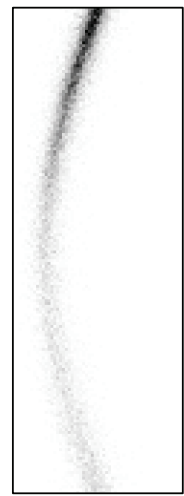

Custom

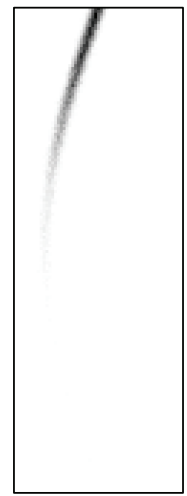

Custom (VR)

Figure 11. Captions of histograms over $\{s, \phi\}$ and single 2D sinogram of voxel simulation located $15 \mathrm{~mm}$ off the axial axis (left) and single $2 \mathrm{D}$ sinogram with $j_{z a}=13$ and $j_{z b}=22$ (right). The 2D sinogram is a subset of a single column of the SM and can be appreciated by the different degree of sparsity between GATE simulation, the custom simulation without VR technique and custom simulation employing a LUT to model the crystal interaction.

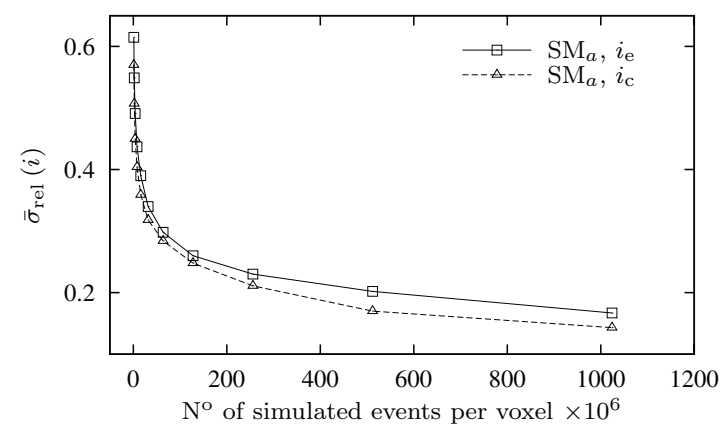

Figure 12. Mean relative error per voxel $\left(\bar{\sigma}_{\text {rel }}(i)\right)$ versus number of simulated events per voxel. Two voxels belonging to the central transaxial slice are compared: $i_{e}$, located in the extreme of the FOV, and $i_{c}$, located in the centre of the FOV. The system matrix $\mathrm{SM}_{a}$ corresponds to a grid of $112 \times 112 \times 112$ voxels, with a voxel size of $0.4 \mathrm{~mm} \times 0.4 \mathrm{~mm} \times 0.4 \mathrm{~mm}$.

\subsection{Point-source phantom}

The measured sensitivity of this study was $58 \mathrm{cps} \mu \mathrm{Ci}^{-1}$, collecting a total amount of $1.05 \times$ $10^{7}$ coincidences with $120 \mathrm{~min}$ of acquisition time. A total number of eight iterations over ten subsets have been performed in all cases, i.e. 80 sub-iterations of the OSEM algorithm. No regularization has been applied. For SSRB and FORE applied before 2D-OSEM, the axial rebinning has been chosen to 15 pixelated crystals. Transaxial and coronal views of reconstructed images with 3D-OSEM and 2D-OSEM methods are shown in figure 14. The 


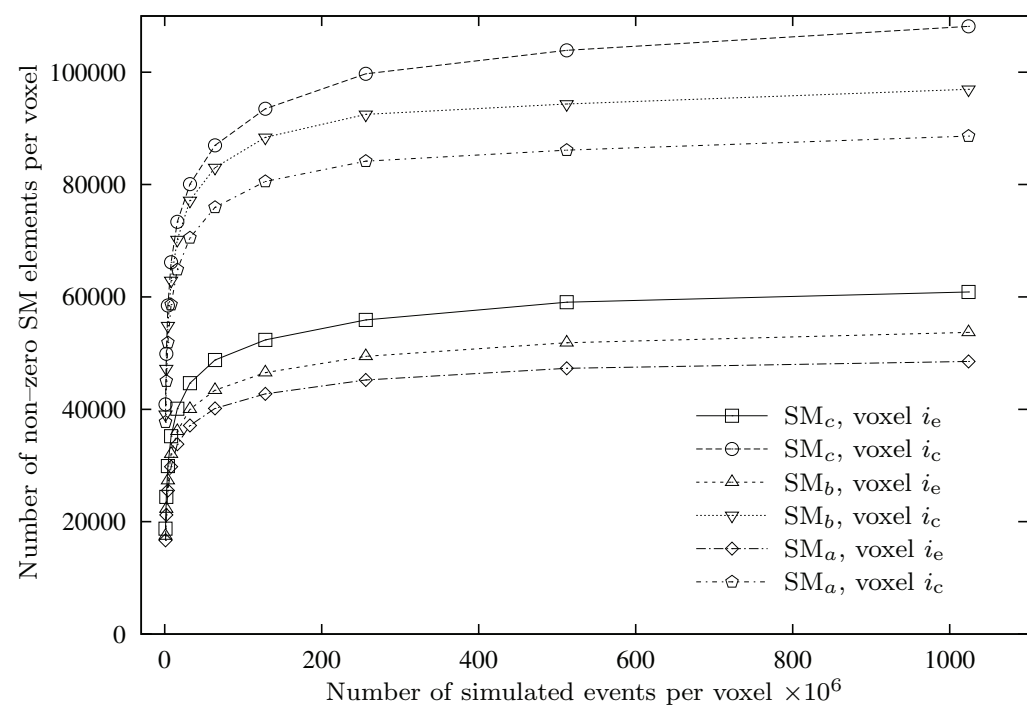

Figure 13. The number of non-zero SM elements $j$ associated with two voxels $i_{c}$ and $i_{e}$, located respectively in the centre and the extreme of the central transaxial slice of the FOV. Three different grid discretizations of the image volume have been studied with dimensions $56 \times 56 \times 56$ voxels $\left(\mathrm{SM}_{c}\right), 112 \times 112 \times 56$ voxels $\left(\mathrm{SM}_{b}\right)$, and $112 \times 112 \times 112$ voxels $\left(\mathrm{SM}_{a}\right)$.
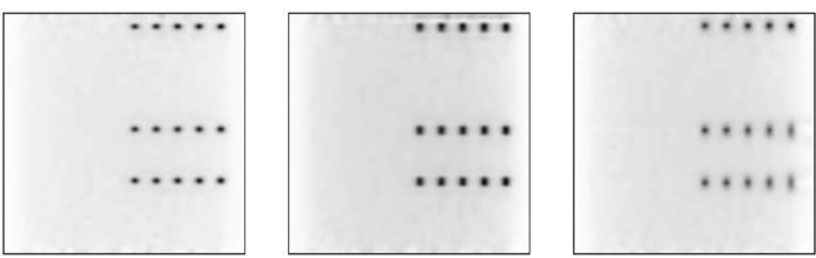

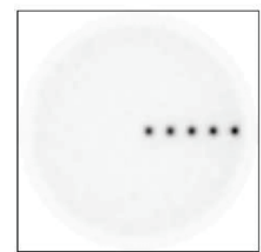

(a) 3D-OSEM, $\mathrm{SM}_{a}$

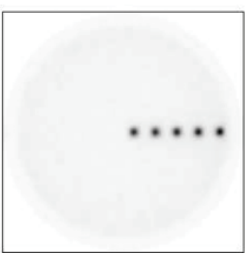

(b) 3D-OSEM, $\mathrm{SM}_{b}$

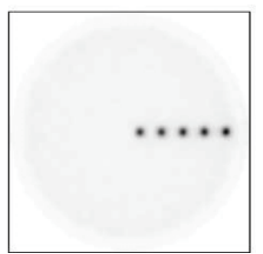

(c) 2D-OSEM+SSRB

Figure 14. Reconstructions of point sources placed in a hot cylinder, simulated with GATE.

SM obtained with the VR method were used in the reconstructions presented in this and the following sections.

In figure 15 the arithmetic mean of radial, tangential and axial resolution, specified by the full width half maximum (FWHM) distance of the adjusted Gaussian curve, has been plotted against the radial position. The points selected correspond to the row situated in the central transaxial plane. 


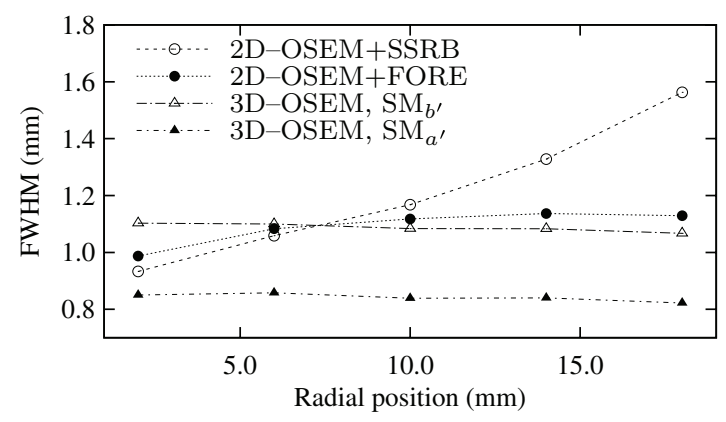

Figure 15. Spatial resolution as function of the radial position for 2D-OSEM and 3D-OSEM reconstruction methods, for the point-source phantom.

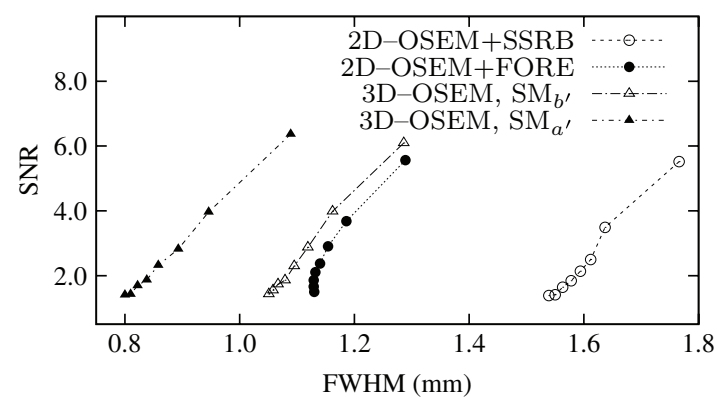

Figure 16. Signal-noise ratio versus resolution trade-off for 2D-OSEM and 3D-OSEM reconstruction methods, evaluated with the point-source phantom.

Noise-resolution curves are plotted in figure 16 from 1 to 8 iterations. The signal-to-noise ratio (SNR) is interpreted as the mean divided by the standard deviation, measured on the warm cylinder over 500 random selected voxels per slice, and FWHM is referred to as the mean value of the activity distribution measured at the point sources in the central transaxial plane. Voxel sizes used in figures 15 and 16 were selected to be $0.2 \mathrm{~mm}$ in the transaxial slice for both $2 \mathrm{D}$ and $3 \mathrm{D}$ reconstructions. For the axial dimension, the $2 \mathrm{D}$ rebinning method imposed a voxel size of $0.8 \mathrm{~mm}$, while for 3D reconstruction the size was of $0.8 \mathrm{~mm}$ for $\mathrm{SM}_{b^{\prime}}$ and $0.4 \mathrm{~mm} \mathrm{SM}_{a^{\prime}}$ corresponding to the single slice and two slices SM simulation option.

\subsection{Derenzo-type phantom}

The sensitivity of this study was $130 \mathrm{cps} \mu \mathrm{Ci}^{-1}$ for the setup with rods parallel to the axial axis and $120 \mathrm{cps} \mu \mathrm{Ci}^{-1}$ for the setup with rods perpendicular to the axial axis. Acquisition time was $33 \mathrm{~min}$ in both configurations. Figures 17 and 19 show reconstructed volumes for the two proposed phantom setups with 2D-OSEM and 3D-OSEM, and with the two distinct $z$ resolutions in the $3 \mathrm{D}$ case. Transaxial and coronal views are shown. The line profiles plotted in figure 18 correspond to volumes of figure 17, crossing the $1.2 \mathrm{~mm}$ and $2.4 \mathrm{~mm}$ rods which are perpendicular to the axial axis. Parameters were fixed to ten iterations with ten balanced subsets each one for all cases. In order to control the noise amplification, a 3D separable Gaussian filter has been applied in each sub-iteration in the regularization scheme, 

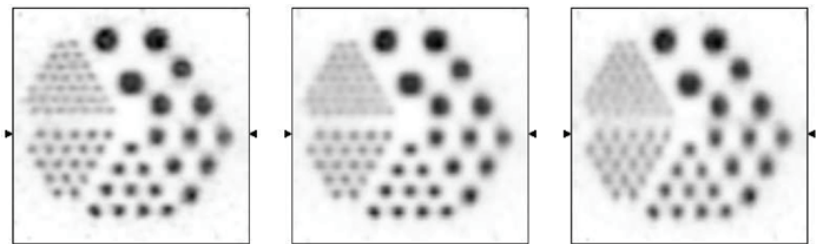

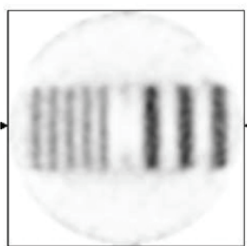

(a) 3D-OSEM, $\mathrm{SM}_{a}$

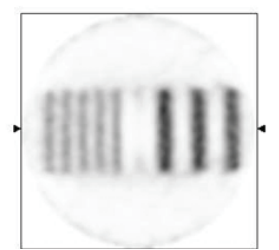

(b) 3D-OSEM, $\mathrm{SM}_{b}$

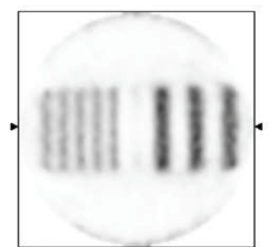

(c) 2D-OSEM+FORE

Figure 17. Reconstructions of a Derenzo-type phantom simulated with GATE, with rods perpendicular to the $z$ axis of the scanner. Coronal and transaxial planes are shown for 3DOSEM (a)-(b) and FORE+2D-OSEM (c) reconstruction schemes. The 3D method has been tested with $\mathrm{SM}_{a}$ and $\mathrm{SM}_{b}$, i.e. grid sizes in $z$ axis of $0.4 \mathrm{~mm}$ (a) and $0.8 \mathrm{~mm}$ (b) respectively. Ten subsets and ten complete EM iterations have been performed in all cases, with MAP regularization.

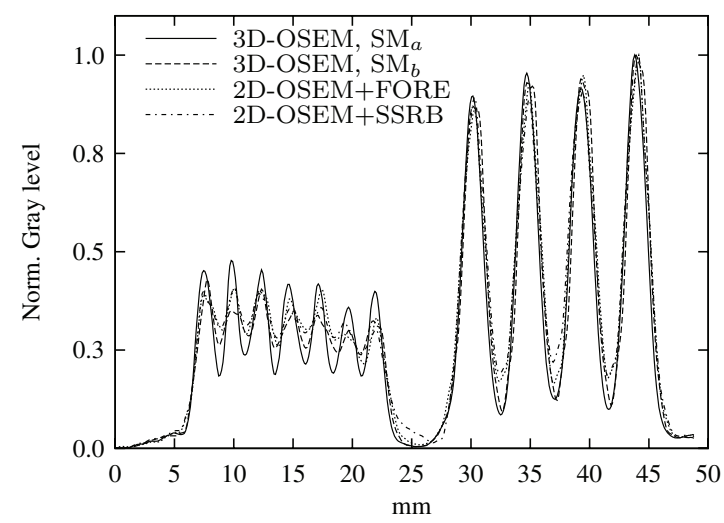

Figure 18. Line profiles across the Derenzo-type phantoms of figure 17, crossing the $1.2 \mathrm{~mm}$ and $2.4 \mathrm{~mm}$ rods which are perpendicular to the axial axis.

with $\sigma=0.5 \mathrm{~mm}$ in the $x, y$ and $z$ directions and $\beta_{r}=0.10$. No scatter or attenuation corrections have been performed.

\subsection{Quality phantom}

The sensitivity of this study was $78 \mathrm{cps} \mu \mathrm{Ci}^{-1}$ and the acquisition time was $30 \mathrm{~min}$. In figure 20 the 2D-OSEM and 3D-OSEM reconstructions of the quality phantom study are shown at different views.

Qualitative results were evaluated over several ROIs defined in hot and cold cylinders and background regions, leaving spacing of $1 \mathrm{~mm}$ with respect to the edges to avoid bias due to 

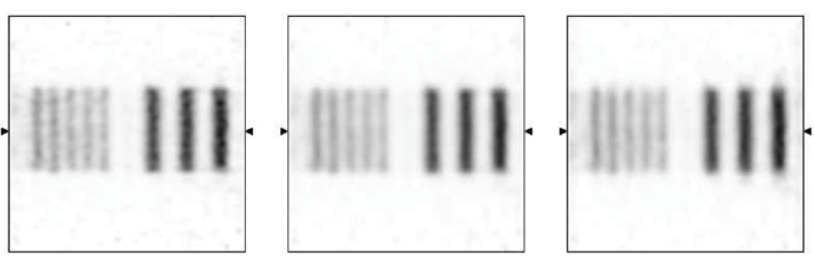

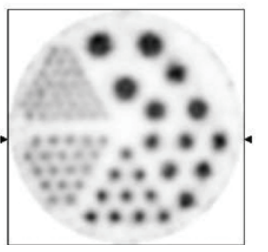

(a) 3D-OSEM, $\mathrm{SM}_{a}$

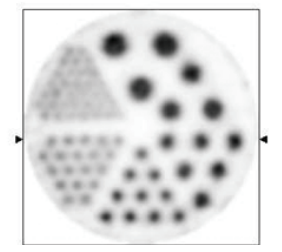

(b) 3D-OSEM, $\mathrm{SM}_{b}$

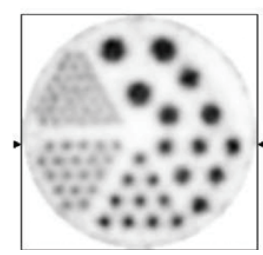

(c) 2D-OSEM+FORE

Figure 19. Reconstructions of a Derenzo-type phantom simulated with GATE, with rods parallel to the $z$ axis of the scanner. Coronal and transaxial planes are shown for 3D-OSEM (a)-(b) and FORE+2D-OSEM (c) reconstruction schemes. $\mathrm{SM}_{a}$ (a) and $\mathrm{SM}_{b}$ (b) resolution grids were employed in 3D methods, which correspond respectively to grid sizes in the $z$ axis of $0.4 \mathrm{~mm}$ and $0.8 \mathrm{~mm}$. Ten subsets and ten complete EM iterations have been performed in all cases, with MAP regularization.
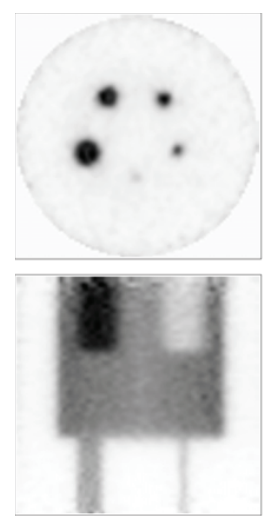

(a) FORE+2D-OSEM
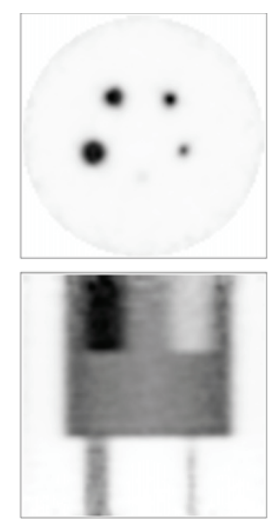

(b) 3D-OSEM,SMa
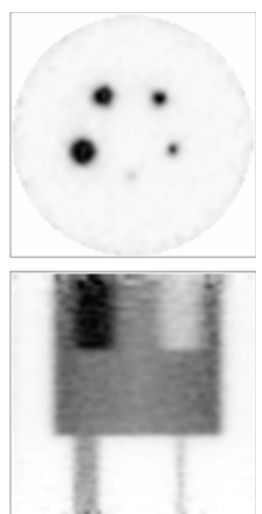

(c) 3D-OSEM,SMb

Figure 20. Reconstructions of the quality phantom simulated with GATE. (a), (b) and (c) Transaxial and coronal sections. (a) FORE+2D-OSEM reconstruction. (b) 3D-OSEM reconstruction. (c) 3D-OSEM reconstruction, with small voxel size. Ten subsets and eight iterations in the three cases.

location misalignments and partial volume errors. Transaxial locations of this ROIs are shown in figure 21. Note that the cold background region is surrounding the small cylinders in the lower part of the phantom.

In figure 22 the per cent contrast recovery for the hot cylinder ROI $\left(Q_{H}\right)$ and cold cylinder ROI $\left(Q_{C}\right)$ are shown for $2 \mathrm{D}$ and $3 \mathrm{D}$ reconstruction modes. $Q_{H}^{\prime}$ figure of merit for small cylinders is shown in figure 23. Cylinder data were measured over ROIs of $10 \mathrm{~mm}$ width in the axial dimension. 


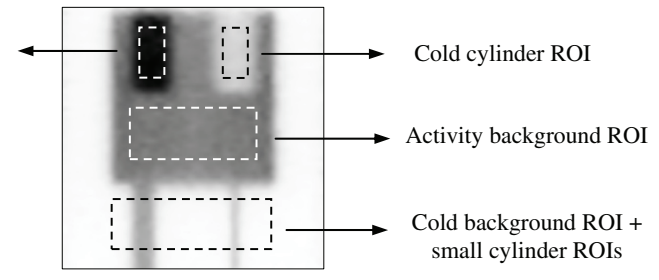

Coronal view

Figure 21. Transaxial view of ROIs defined in the quality phantom.
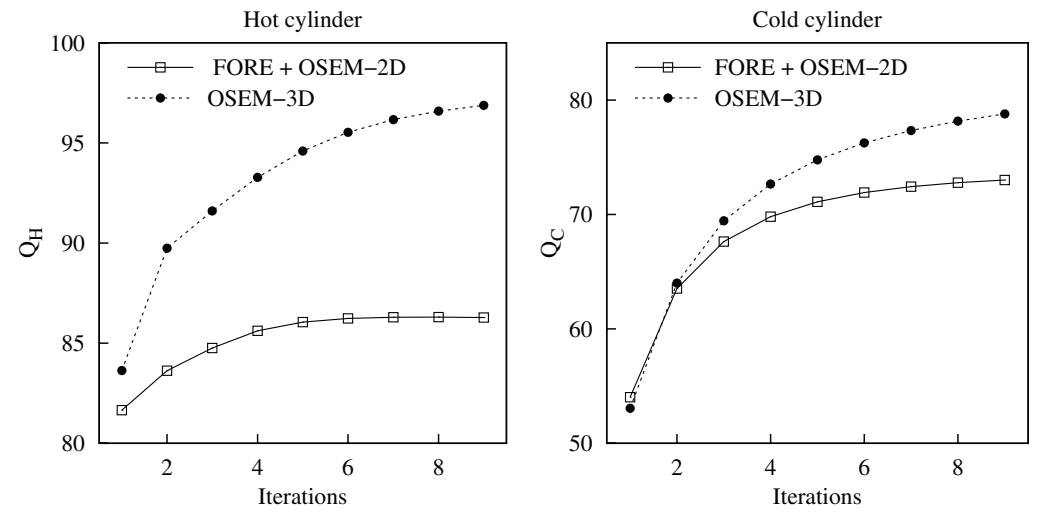

Figure 22. Per cent contrast recovery for the hot cylinder and cold cylinder and its evolution according to the number of iterations over the whole set of subsets of OSEM. Ten subsets were used in $2 \mathrm{D}$ and $3 \mathrm{D}$ reconstruction methods.
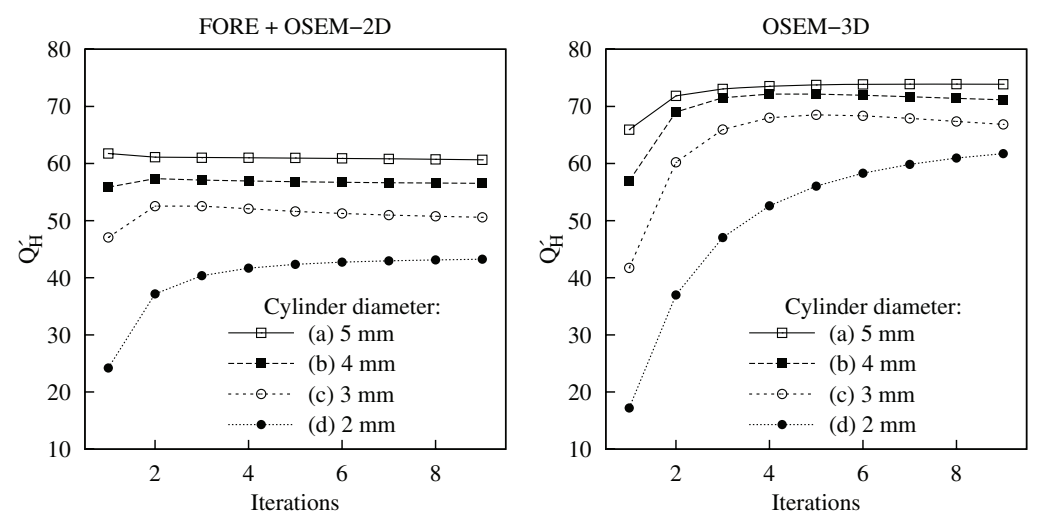

Figure 23. Per cent contrast recovery of small cylinders and its evolution according to the number of iterations for FORE+2D-OSEM and 3D-OSEM methods. Ten subsets have been employed in both cases. 

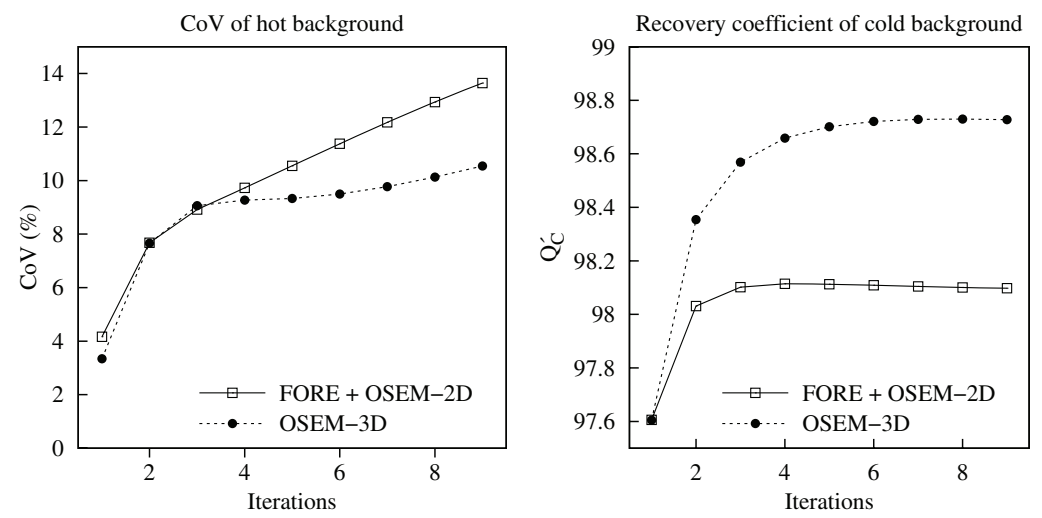

Figure 24. $\mathrm{CoV}$ (in percentage) of hot background ROI and $Q_{C}$ contrast recovery of cold background ROI. Ten subsets have been employed with FORE+2D-OSEM and 3D-OSEM methods.

The last qualitative measurements over the quality phantom, plotted in figure 24, compare the coefficient of variance $(\mathrm{CoV})$ (defined as the standard deviation divided by the mean) of the hot background ROI (see figure 21), as well as the mean activity value of the cold background ROI, relative to the hot background ROI.

\section{Discussion and conclusions}

Considering the SM custom simulation, figures 10 and 11 show a very good agreement between the results obtained by our method and GATE simulations, while the proposed methodology requires a small fraction of computation time. The comparison was performed over two voxels located respectively in the centre of the FOV and $15 \mathrm{~mm}$ off the axial axis in the central transaxial plane. Histogrammed coincidences assigned to oblique sinograms showed similar profile, while the VR method reduced the degree of sparsity of the SM limiting the non-zero bins due to inter-crystal scatter. The value of $\bar{\sigma}_{\text {rel }}$ associated with representative voxels was used to select the number of simulated events required for SM modelling.

The transaxial views in figure 14 exhibit similar spatial resolution for both $3 \mathrm{D}$ and $2 \mathrm{D}$ methods as the voxel size is the same for all methods in $x$ and $y$ dimensions, and 2D rebinning methods project LORs without errors in these dimensions. On the other hand, coronal views in figure 14 demonstrate higher resolution when $\mathrm{SM}_{a}$ is employed. SSRB+2D-OSEM show worse axial spatial resolution results in the extremes of the FOV, although this effect is less significant in the upper row of the point sources because the maximum value of axial rebinning has not been reached.

The quantitative results of figure 15 confirm the visual evidence of figure 14, and a submillimetre spatial resolution has been achieved by $3 \mathrm{D}$ reconstruction with $\mathrm{SM}_{a}$ which clearly outperforms the reconstruction with the FORE+2D-OSEM method.

Noise-resolution curves in figure 16 show a superior performance of 3D-OSEM with $\mathrm{SM}_{a}$ in terms of spatial resolution due to a better sampling in $z$. This method outperforms 3D reconstructions with $\mathrm{SM}_{b}$ as well as the $2 \mathrm{D}$ method.

In the line profiles shown in figure 18 , the highest $z$ resolution is obtained with the $3 \mathrm{D}$ reconstruction method with the smallest $\mathrm{SM}$ voxel size of $0.4 \mathrm{~mm}$ in the $z$ direction. The $3 \mathrm{D}$ reconstructed images with voxel size of $0.8 \mathrm{~mm}$ in the $z$ direction have an intermediate resolution, while the $2 \mathrm{D}$ case gets worse specially in rods far from the centre of the FOV. 
In figure 19, where the Derenzo-type phantom is positioned with the active rods parallel to the axial axis of the scanner, no difference is appreciated between resolution recovery in $2 \mathrm{D}$ and $3 \mathrm{D}$ reconstruction methods since the voxel size in the $x-y$ plane is fixed to $0.4 \mathrm{~mm}$ in all cases. Furthermore, the rod alignment along the $z$ direction of the scanner guarantees that the rebinning process does not degrade the resolution in the transaxial plane, except at the rod extremes.

In figure 20 (quality phantom study), the improvement in spatial resolution of the 3D method with a high-resolution SM can be observed in the axial direction along the edges between regions with different activity concentrations.

Data shown in figure 22 demonstrate that recovery coefficients are better in 3D modes. The improvement is more significant in measurements performed in the hot cylinder ROI, while the cold cylinder ROI contrast recovery gives poorer results due to the lack of scatter and attenuation corrections.

From figure 23 it can be noted that the highest cylinder contrast is obtained for all cases with the $3 \mathrm{D}$ reconstruction mode, although in the first iterations, the $2 \mathrm{D}$ reconstruction mode has a higher recovery coefficient.

$\mathrm{CoV}$ and recovery coefficient measurements over the quality phantom (figure 24) show better contrast in $3 \mathrm{D}$ reconstruction modes except during early iterations, where $\mathrm{CoV}$ and relative mean ratios are similar in 2D-OSEM. In latter iterations, CoV values are greater in 2D-OSEM due to the characteristic noise amplification in unregularized OSEM reconstruction, while the mean average ratio of cold and hot background regions remain stable at values nearly two times smaller.

A fast fully 3D-OSEM reconstruction algorithm has been developed, implemented and evaluated for a high-resolution rotating head PET scanner composed of two pairs of opposed planar pixelated blocks. The proposed scheme employs a pre-calculated SM based on MC methods, efficiently stored with sparse matrix format. Rotation and reflection symmetries in the transaxial plane are compatible with the simulated SM matrix and allow us to model only the eighth part of voxels in a transaxial plane. Axial redundancies and reflection symmetries, combined with the shifted axial grid alignment imply that only two transaxial planes need to be simulated for the finest grid resolution. Total simulation time is maintained below $100 \mathrm{~h}$ in this case.

The full SM can be loaded in RAM in a standard PC, speeding-up the reconstruction process. We use a model of the scanner which incorporates crystal attenuation and scatter effects.

2D-OSEM has also been implemented with a post-processing of the 3D SM. Experiments have shown that these fast methods exhibit similar resolution to the equivalent $3 \mathrm{D}$ ones in the transaxial plane, being surpassed in measurements related to the axial axis.

The experiments performed with simulated phantoms show that the 3D-OSEM algorithm improves resolution when compared to 2D-OSEM reconstruction methods when voxel size in the $z$ dimension is fixed to one-quarter the length of the pixelated crystal row.

\section{Acknowledgments}

This work has been partially supported by the CDTEAM project, CENIT programme (Spanish Ministry of Industry) and EMIL (EU Network of Excellence).

CIBER-BBN is an initiative funded by the VI National R\&D\&I Plan 2008-2011, Iniciativa Ingenio 2010, Consolider Program, CIBER Actions and financed by the Instituto de Salud Carlos III with assistance from the European Regional Development Fund. 


\section{References}

Alenius S and Ruotsalainen U 2002 Generalization of median root prior reconstruction IEEE Trans. Med. Imaging 21 1413-20

Alenius S, Ruotsalainen U and Astola J 1998 Using local median as the location of the prior distribution in iterative emission tomography image reconstruction IEEE Trans. Nucl. Sci. 45 3097-104

Alessio A M, Kinahan P E and Lewellen T K 2006 Modeling and incorporation of system response functions in 3-D whole body PET IEEE Trans. Med. Imaging 25 828-37

Benlloch J M et al 2007 Scanner calibration of a small animal PET camera based on continuous LSO crystals and flat panel PSPMTs Nucl. Instrum. Methods A $57126-9$

Berger M J, Hubbell J H, Seltzer S M, Chang J, Coursey J S, Sukumar R and Zucker D S 1998 XCOM: photon cross sections database NIST Standard Reference Database http://physics.nist.gov/PhysRefData/Xcom/Text/XCOM.html

Chatziioannou A, Qi J, Moore A, Annala A, Nguyen K, Leahy R and Cherry S R 2000 Comparison of 3-D maximum a posteriori and filtered backprojection algorithms for high-resolution animal imaging with microPET IEEE Trans. Med. Imaging 19 507-12

Colsher J G 1980 Fully 3-dimensional positron emission tomography Phys. Med. Biol. 25 103-15

Daube-Witherspoon M E and Muehllehner G 1987 Treatment of axial data in 3-dimensional PET J. Nucl. Med. 28 1717-24

de Benedetti S, Cowan C E, Konneker W R and Primakoff H 1950 On the angular distribution of 2-photon annihilation radiation Phys. Rev. 77 205-12

de la Prieta R, Hernandez J A, Schiavi E and Malpica N 2006 Analytical geometric model for photon coincidence detection in 3D IEEE Nucl. Sci. Symp. Med. Imaging Conf. 4 2229-32

Defrise M, Kinahan P E, Townsend D W, Michel C, Sibomana M and Newport D 1997 Exact and approximate rebinning algorithms for 3-D PET data IEEE Trans. Med. Imaging 16 145-58

Del Guerra A et al 2006 Performance evaluation of the fully engineered YAP-(S)PET scanner for small animal imaging IEEE Trans. Nucl. Sci. 53 1078-83

España S, Herráiz J L, Vicente E, Vaquero J J, Desco M and Udías J M 2009 PeneloPET, a Monte Carlo PET simulation tool based on PENELOPE: features and validation Phys. Med. Biol. 54 1723-42

Fessler J A and Hero A O 1995 Penalized maximum-likelihood image-reconstruction using space-alternating generalized EM algorithms IEEE Trans. Image Process. 4 1417-29

Frese T, Rouze N C, Bouman C A, Sauer K and Hutchins G D 2003 Quantitative comparison of FBP, EM, and Bayesian reconstruction algorithms for the IndyPET scanner IEEE Trans. Med. Imaging 22 258-76

Gimenez E N, Nacher E, Gimenez M, Benlloch J M and Rafecas M 2006 Comparison of different approaches based on Monte Carlo methods to calculate the system matrix for small animal PET Nucl. Instrum. Methods A 569 346-9

Green P J 1990 Bayesian reconstructions from emission tomography data using a modified EM algorithm IEEE Trans. Med. Imaging 9 84-93

Herman G T and Meyer L B 1993 Algebraic reconstruction techniques can be made computationally efficient IEEE Trans. Med. Imaging 12 600-9

Herráiz J L, España S, Vaquero J J, Desco M and Udías J M 2006 FIRST: fast iterative reconstruction software for (PET) tomography Phys. Med. Biol. 51 4547-65

Herrero V, Colom R, Gadea R, Lerche C W, Cerda J, Sebastia A and Benlloch J M 2007 Front-end circuit for position sensitive silicon and vacuum tube photomultipliers with gain control and depth of interaction measurement Nucl. Instrum. Methods A 576 118-22

Hua X M 1997 Monte Carlo simulation of comptonization in inhomogeneous media Comput. Phys. 11 660-8

Hudson H M and Larkin R S 1994 Accelerated image-reconstruction using ordered subsets of projection data IEEE Trans. Med. Imaging 13 601-9

Huisman M C, Reder S, Weber A W, Ziegler S I and Schwaiger M 2007 Performance evaluation of the Philips MOSAIC small animal PET scanner Eur. J. Nucl. Med. Mol. Imaging 34 532-40

Ishii K et al 2007 First achievement of less than $1 \mathrm{~mm}$ FWHM resolution in practical semiconductor animal PET scanner Nucl. Instrum. Methods A 576 435-40

Jacobson M et al 2000 Enhanced 3D PET OSEM reconstruction using inter-update Metz filtering Phys. Med. Biol. 45 2417-39

Jan S et al 2004 GATE: a simulation toolkit for PET and SPECT Phys. Med. Biol. $494543-61$

Jeavons A P, Chandler R A and Dettmar C A R 1999 A 3D HIDAC-PET camera with sub-millimetre resolution for imaging small animals IEEE Trans. Nucl. Sci. 46 468-73

Johnson C A, Seidel J, Carson R E, Gandler W R, Sofer A, Green M V and DaubeWitherspoon M E 1997 Evaluation of 3D reconstruction algorithms for a small animal PET camera IEEE Trans. Nucl. Sci. 44 1303-8

Johnson C A, Yan Y C, Carson R E, Martino R L and Daube-Witherspoon M E 1995 A system for the 3D reconstruction of retracted-septa PET data using the EM algorithm IEEE Trans. Nucl. Sci. 42 1223-7 
Joung J, Miyaoka R S and Lewellen T K 2002 cMiCE: a high resolution animal PET using continuous LSO with a statistics based positioning scheme Nucl. Instrum. Methods A $489584-98$

Kinahan P E and Rogers J G 1989 Analytic 3D image-reconstruction using all detected events IEEE Trans. Nucl. Sci. 36 964-8

Kontaxakis G, Strauss L G, Thireou T, Ledesma-Carbayo M J, Santos A, Pavlopoulos S A and DimitrakopoulouStrauss A 2002 Iterative image reconstruction for clinical PET using ordered subsets, median root prior and a web based interface Mol. Imaging Biol. 4 219-31

Lage E, Vaquero J J, Sisniega A, España S, Tapias G, Abella M, Rodriguez-Ruano A, Ortuño J E, Udias A and Desco M 2009 Phys. Med. Biol. 54 5427-41

Lazaro D, Bitar ZE, Breton V, Hill D and Buvat I 2005 Design and performance evaluation of a coplanar multimodality scanner for rodent imaging Phys. Med. Biol. $503739-54$

Leahy R M and Qi J Y 2000 Statistical approaches in quantitative positron emission tomography Stat. Comput. 10 147-65

Lecomte R 2004 Technology challenges in small animal PET imaging Nucl. Instrum. Methods A 527 157-65

Levin C S and Hoffman E J 1999 Calculation of positron range and its effect on the fundamental limit of positron emission tomography system spatial resolution Phys. Med. Biol. 44 781-99

Lewellen T K, Harrison R L and Vannoy S 1998 The SIMSET Program Monte Carlo Calculations in Nuclear Medicine (London: Institute of Physics Publishing)

McElroy D P, Pimpl W, Pichler B J, Rafecas M, Schuler T and Ziegler S I 2005 Characterization and readout of MADPET II detector modules: validation of a unique design concept for high resolution small animal PET IEEE Trans. Nucl. Sci. 52 199-204

Mesina C T, Boellaard R, Jongbloed G, van der Vaart A W and Lammertsma A A 2003 Experimental evaluation of iterative reconstruction versus filtered back projection for 3D [O-15] water PET activation studies using statistical parametric mapping analysis Neuroimage 19 1170-9

Moehrs S, Defrise M, Belcari N, Del Guerra A, Bartoli A, Fabri S and Zanetti G 2008 Multi-ray-based system matrix generation for 3D PET reconstruction Phys. Med. Biol. 53 6925-45

NEMA 2007 Performance measurements of positron emission tomographs NEMA Standards Publication NU 4-2007 Technical Report (Rosslyn, VA: National Electrical Manufacturers Association)

NEMA 2008 Performance measurements for small animal positron emission tomographs NEMA Standards Publication NU 4-2008 Technical Report (Rosslyn, VA: National Electrical Manufacturers Association)

Ortuño J E, Guerra P, Rubio J L, Kontaxakis G and Santos A 2006 3D OSEM-based iterative image reconstruction for high resolution PET using precalculated system matrix Nucl. Instrum. Methods A $569440-4$

Ortuño J E, Kontaxakis G, Guerra P and Santos A 2004 3D-OSEM transition matrix for high resolution PET imaging with modeling of the gamma-event detection IEEE Nucl. Sci. Symp. Med. Imaging Conf. 5 3046-50

Ortuño J E, Vaquero J J, Kontaxakis G, Desco M and Santos A 2003 Preliminary studies on the design and simulation of high resolution small animal PET scanners with octagonal geometry IEEE Nucl. Sci. Symp. Med. Imaging Conf. $32053-7$

Panin V Y, Kehren F, Rothfuss H, Hu D, Michel C and Casey M E 2006 PET reconstruction with system matrix derived from point source measurements IEEE Trans. Nucl. Sci. 53 152-9

Qi J Y, Leahy R M, Cherry S R, Chatziioannou A and Farquhar T H 1998 High-resolution 3D Bayesian image reconstruction using the MicroPET small-animal scanner Phys. Med. Biol. 43 1001-13

Rafecas M, Mosler B, Dietz M, Pogl M, Stamatakis A, McElroy D P and Ziegler S I 2004 Use of a Monte Carlo-based probability matrix for 3-D iterative reconstruction of MADPET II data IEEE Trans. Nucl. Sci. 51 2597-605

Raheja A, Doniere T F and Dhawan A P 1999 Multiresolution expectation maximization reconstruction algorithm for positron emission tomography using wavelet processing IEEE Trans. Nucl. Sci. 46 594-602

Ranganath M V, Dhawan A P and Mullani N 1988 A multigrid expectation maximization reconstruction algorithm for positron emission tomography IEEE Trans. Med. Imaging 7 273-8

Rannou F R and Chatziioannou A F 2004 Fully 3D system model estimation of OPET by Monte Carlo simulation IEEE Nucl. Sci. Symp. Med. Imaging Conf. $63433-6$

Rouze N C and Hutchins G D 2003 Design and characterization of IndyPET II: a high-resolution, high-sensitivity dedicated research scanner IEEE Trans. Nucl. Sci. 50 1491-7

Schafers K P, Reader A J, Kriens M, Knoess C, Schober O and Schafers M 2005 Performance evaluation of the 32-module quadHIDAC small-animal PET scanner J. Nucl. Med. 46 996-1004

Scheins J J, Boschen F and Herzog H 2006 Analytical calculation of volumes-of-intersection for iterative, fully 3-D PET reconstruction IEEE Trans. Med. Imaging 25 1363-9

Selivanov V V, Picard Y, Cadorette J, Rodrigue S and Lecomte R 2000 Detector response models for statistical iterative image reconstruction in high resolution PET IEEE Trans. Nucl. Sci. 47 1168-75

Shepp L A and Vardi Y 1982 Maximum likelihood reconstruction in positron emission tomography IEEE Trans. Med. Imaging 1 113-22 
Shokouhi S et al 2004 Statistical 3D image reconstruction for the RatCAP PET tomograph using a physically accurate, Monte Carlo based system matrix IEEE Nucl. Sci. Symp. Med. Imaging Conf. 6 3901-5

Siddon R L 1985 Fast calculation of the exact radiological path for a 3-dimensional CT Array Med. Phys. 12 252-5

Strul D, Slates R B, Dahlbom M, Cherry S R and Marsden P K 2003 An improved analytical detector response function model for multilayer small-diameter PET scanners Phys. Med. Biol. 48 979-94

Surti S, Karp J S, Perkins A E, Cardi C A, Daube-Witherspoon M E, Kuhn A and Muehllehner G 2005 Imaging performance of A-PET: a small animal PET camera IEEE Trans. Med. Imaging 24 844-52

Tai Y C, Ruangma A, Rowland D, Siegel S, Newport D F, Chow P L and Laforest R 2005 Performance evaluation of the MicroPET Focus: a third-generation MicroPET scanner dedicated to animal imaging J. Nucl. Med. 46 455-63

Terstegge A, Weber S, Herzog H, Muller-Gartner H W and Halling H 1997 High resolution and better quantification by tube of response modelling in 3D PET reconstruction IEEE Nucl. Sci. Symp. Med. Imaging Conf. 3 1603-7

Tohme M S and Qi J Y 2009 Iterative image reconstruction for positron emission tomography based on a detector response function estimated from point source measurements Phys. Med. Biol. 54 3709-25

Tsuda T, Murayama H, Kitamura K, Inadama N, Yamaya T, Yoshida E, Nishikido F, Hamamoto M, Kawai H and Ono Y 2006 Performance evaluation of a subset of a four-layer LSO detector for a small animal DOI PET scanner: jPET-RD IEEE Trans. Nucl. Sci. 53 35-9

Vaquero J J, Lage E, Redondo S, Abella M, Pascau J, Sanchez J, Vicente E, Soto-Montenegro M L and Desco M 2005a Co-planar PET/CT for small animal imaging IEEE Nucl. Sci. Symp. Med. Imaging Conf. 3 1748-51

Vaquero J J, Lage E, Ricon L, Abella M, Vicente E and Desco M 2005b rPET detectors design and data processing IEEE Nucl. Sci. Symp. Med. Imaging Conf. 5 2885-9

Vaquero J J, Molins A, Ortuño J E, Pascau J and Desco M 2004 Preliminary results of the small animal rotational positron emission tomography scanner Mol. Imaging Biol. 6102

Wang Y C, Seidel J, Tsui B M W, Vaquero J J and Pomper M G 2006 Performance evaluation of the GE Healthcare eXplore VISTA dual-ring small-animal PET scanner J. Nucl. Med. 47 1891-900

Weber S, Morel C, Simon L, Krieguer M, Rey M, Gundlich B and Khodaverdi M 2006 Image reconstruction for the ClearPET (TM) Neuro Nucl. Instrum. Methods A 569 381-5

Yamaya T et al 2005 Transaxial system models for jPET-D4 image reconstruction Phys. Med. Biol. 50 5339-55

Yamaya T, Yoshida E, Obi T, Ito H, Yoshikawa K and Murayama H 2008 First human brain imaging by the jPET-D4 prototype, with a pre-computed system matrix IEEE Trans. Nucl. Sci. 55 2482-92

Ziemons K et al 2005 The ClearPET (TM) project: development of a 2nd generation high-performance small animal PET scanner Nucl. Instrum. Methods A 537 307-11 